L-edge-magnon

\title{
Effect of broken symmetry on resonant inelastic x-ray scattering from undoped cuprates
}

\author{
Jun-ichi Igarashi \\ Faculty of Science, Ibaraki University, Mito, Ibaraki 310-8512, Japan \\ Tatsuya Nagao \\ Faculty of Engineering, Gunma University, Kiryu, Gunma 376-8515, Japan
}

(Dated: October 15, 2018)

\begin{abstract}
We study the magnetic excitation spectra of resonant inelastic x-ray scattering (RIXS) at the $L$-edge from undoped cuprates beyond the fast collision approximation. We analyse the effect of the symmetry breaking ground state on the RIXS process of the Heisenberg model by using a projection procedure. We derive the expressions of the scattering amplitude in both one-magnon and twomagnon excitation channels. Each of them consists of the isotropic and anisotropic contributions. The latter is a new finding and attributed to the long range order of the ground state. The presence of anisotropic terms is supported by numerical calculations on a two-dimensional spin cluster. We express the RIXS spectra in the form of spin-correlation functions with the coefficients evaluated on the cluster, and calculate the function in a two dimensional system within the 1/S expansion. Due to the anisotropic terms, the spectral intensities are considerably enhanced around momentum transfer $\mathbf{q}=0$ in both one-magnon and two-magnon excitation channels. This finding may be experimentally confirmed by examining carefully the q-dependence of the spectra.
\end{abstract}

PACS numbers:

\section{INTRODUCTION}

Resonant inelastic x-ray scattering (RIXS) has attracted much interest as a useful tool to investigate excited states in solids ${ }^{1}$. The L-edge RIXS experiments have been recently carried out with high energy resolution in transition-metal compounds, which have revealed magnetic excitations as spectral peaks in the low-energy region ${ }^{2}-4$. Starting from the undoped cuprates, the activity spreads over, rapidly and widely, doped high- $\mathrm{T}_{\mathrm{c}}$ cuprates $^{-\frac{5}{-}}$, nickelates ${ }^{8}$, pnictides ${ }^{9}, 5 d$ transition metal compounds ${ }^{10,11}$, and so on. Among them, the investigation on cuprates is one of the most active fields due to the relation with high- $\mathrm{T}_{\mathrm{c}}$ superconductivity. Stimulated by these experiments, theoretical efforts to elucidate mechanism of the magnetic RIXS in cuprates also have developed ${ }^{12-18}$. But rich information involved in the $L$-edge RIXS data such as momentum and energy transfer dependence as well as polarization dependence never ceases to require further reliable and convincing theories more than ever.

The $L$-edge resonance in undoped cuprates is described by the second-order dipole allowed process that a $2 p$-core electron is prompted to an empty $x^{2}-y^{2}$ orbital by absorbing photon and then an occupied $3 d$ electron combines with the core hole by emitting photon. When the $3 d$ orbital in the photo-emitting process is different from the one in the photo-absorbing process, the excitations within the $3 d$ orbitals are brought about, which are called as d-d excitation 19 . When the $3 d$ orbitals in the photoabsorbing and photo-emitting processes are the same $x^{2}-y^{2}$ orbital but their spins are different, magnetic excitations with spin flip could be generated 20,21 . Even if the spins are the same, the spin-conserving excitations could be brought about by the presence of the core hole during its finite lifetime $12-14$. This process could be described only when it is treated beyond the fast collision approximation (FCA) that no relaxation could take place in the intermediate state because of the short lifetime 20,21 .

In our previous papers ${ }^{12,13}$, we have analysed the process leading to the final states in the second-order process, and have clarified how the spin excitations are taken place around the core hole site beyond the FCA. In one dimension, the analysis has been straightforward, since the spherical symmetry in spin space remains intact in the ground state, while in two dimensions under the antiferromagnetic ordered phase, the analysis has been rather complicated due to the breaking of spherical symmetry. In both cases, we have obtained the scattering amplitudes in an invariant form with respect to the polarization vectors of the incident and scattered x-rays, and spin operators. Disregarding possible effects of the symmetry breaking ground state, we have obtained spin excitations extending to neighbours of the core hole site. Such excitations have been clearly observed in a onedimensional system $\mathrm{CaCu}_{2} \mathrm{O}_{3}{ }^{22}$ and in two-dimensional systems $\mathrm{Sr}_{2} \mathrm{CuO}_{2} \mathrm{Cl}_{2} \frac{4}{}$ and $\mathrm{La}_{2} \mathrm{CuO}_{4}{ }^{23}$.

However, under the presence of the antiferromagnetic long-range order, it may be reasonable to presume that the scattering amplitudes include anisotropic terms associated with the direction of the staggered moment, since the second-order process could be affected by the anisotropy originated from the broken symmetry of the ground state. This observation contrasts to neutron scattering, in which the scattering amplitude is directly described by the interaction Hamiltonian between the spins of neutron and electron. The purpose of this paper is to clarify the presence of anisotropic terms in the scatter- 
ing amplitude under the presence of the spin long range order by analysing the second-order process on a model of undoped cuprates, where the low-lying excitations are described by the Heisenberg model. In the scattering amplitudes summarised in an invariant form, we obtain the anisotropic terms, which include a vector characterizing the staggered moment.

To estimate quantitative impact of the anisotropic terms, we evaluate them by carrying out numerical analysis on spin clusters. For a cluster of 13 spins, which is regarded as a model of two-dimensional cuprate, various terms in the scattering amplitudes are calculated. We verify the anisotropic terms have finite contributions. If the connection of the anisotropic terms with the symmetry breaking of the ground state is intrinsic, the weights of anisotropic terms are expected to increase with increasing antiferromagnetic long-range order parameter. This anticipation is confirmed by the numerical calculation on a ring of 12 spins with varying the external staggered magnetic field, which is given in Appendix C.

Collecting up such amplitudes from all the $\mathrm{Cu}$ sites, we derive the RIXS spectra represented by spin correlation functions. When we investigate the correlation functions, analysis on a larger system might be preferable. This is because the spin excitations propagate through the entire crystal in the final state. Thus, we employ the $1 / S$ expansion to the spin operators ${ }^{24}$, which practically enables us to treat an infinite system. As a result, we can express the RIXS spectra in terms of the correlation functions of one-magnon and two-magnon contributions. Since two magnons are excited close to each other, their mutual interaction is important. We treat multiple scattering of two magnons by following the method previously developed 12 . It turns out that the correlation functions for both one-magnon and two-magnon channels have anisotropic contributions in addition to isotropic ones. We find that the anisotropic terms produce substantial enhancement on the RIXS intensities for momentum transfer $\mathbf{q}$ close to the $\Gamma$ point in both channels. This shows a sharp contrast to the fact that the contributions from the isotropic terms vanish at $\mathbf{q}=0$ in both channels. Experimentally, polarization analysis may help to clarify the existence of the anisotropic terms, since the polarization dependence is completely different between the one-magnon and the two-magnon spectra.

The present paper is organized as follows. In section III we describe the second-order dipole allowed process responsible for RIXS process. In section III, we analyse the RIXS process paying attention to the influence of the symmetry breaking on the scattering amplitude, in which anisotropic terms are derived in an invariant form. In section IV we evaluate numerically the amplitudes of creating excitations on a finite size of twodimensional cluster under a molecular field on the boundary. In section $\mathrm{V}$, we derive the RIXS spectra in terms of the spin-correlation functions, which are treated with the $1 / S$ expansion to spin operators. The RIXS spectra consisting of one-magnon and two-magnon excitations are

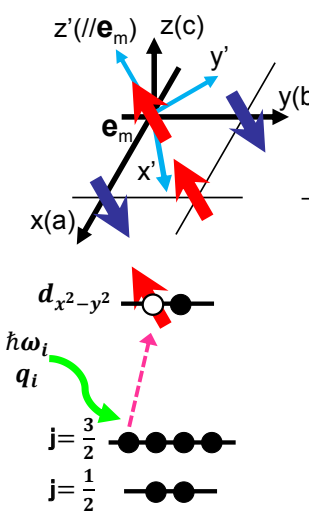

(a)
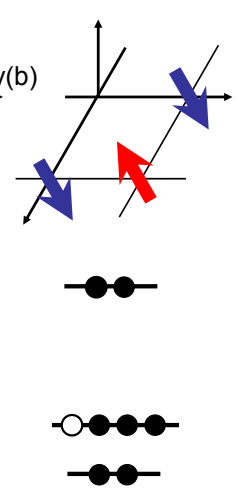

(b)
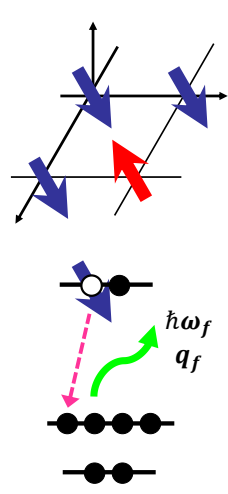

(c)
FIG. 1: A schematic illustration of the RIXS process at the $L_{3}$-edge of cuprates. Filled and open circles stand for the states occupied by electrons and holes, respectively. Red and blue arrows denote the magnetic moments of $3 d$ holes. Wavy green and pink dotted arrows represent the photon and the transitions, respectively. (a) An incident photon excites a core $2 p_{\frac{3}{2}}$ electron into the empty $3 d$ state. The site of the excited electron is chosen as the origin of the crystal-fixed coordinate system with $x, y$, and $z$ axes (or also called as $a, b$, and $c$ axes). The direction of the unit vector of the staggered magnetic moment at the origin is called as $\mathbf{e}_{m}$, which defines the spin coordinate system with $x^{\prime}, y^{\prime}$, and $z^{\prime}$ axes. The spin quantization axis is coincident with $z^{\prime}$ axis. (b) In the intermediate state, spin degree of freedom vanishes at the core-hole site. (c) The excited electron recombines with the core-hole by emitting the scattered photon.

calculated. Section VI is devoted to the concluding remarks. In Appendix A, absorption coefficients at the $L_{2}-$ and $L_{3}$-edges are briefly discussed. A short comment on the projection procedure is given in Appendix B In Appendix [C, we show how the expansion coefficients develop with increasing the staggered moment in a finite-size ring under the external staggered field. Appendix $\mathrm{D}$ outlines the $1 / S$ expansion in the Heisenberg model.

\section{SECOND-ORDER DIPOLE ALLOWED PROCESS}

We briefly explain $L$-edge RIXS in cuprates (See figure 11). The RIXS process at the copper $L$-edge may be described by the electric dipole $(E 1)$ transition between the $2 p$-core states and the $3 d$ states. The $2 p$ states are split into two levels with the total angular momentum $j=\frac{1}{2}$ and $\frac{3}{2}$, which are discriminated as $L_{2}$ and $L_{3}$ edges, respectively, due to the strong spin-orbit interaction. Because each $\mathrm{Cu}$ atom has one hole in the $x^{2}-y^{2}$ orbital in undoped cuprates such as $\mathrm{La}_{2} \mathrm{CuO}_{4}$ and $\mathrm{Sr}_{2} \mathrm{CuCl}_{2} \mathrm{O}_{2}$, we employ a hole picture. Then, the $E_{1}$ transition may be expressed by the interaction between photon and hole 
as,

$$
H_{\text {int }}=w \sum_{\mathbf{q}, \alpha, i, m, \sigma} \frac{D^{\alpha}(j m, \sigma)}{\sqrt{2 \omega_{\mathbf{q}}}} h_{i, j m}^{\dagger} c_{\mathbf{q} \alpha} d_{i \sigma} \mathrm{e}^{\mathrm{i} \mathbf{q} \cdot \mathbf{r}_{i}}+\text { H.c. }
$$

where $c_{\mathbf{q} \alpha}$ annihilates a photon with four-vector $q \equiv$ $\left(\mathbf{q}, \omega_{\mathbf{q}}\right)$ and polarization $\alpha$. The $h_{i, j m}^{\dagger}$ represents the creation operator of the $2 p$ hole with $j m$ at site $i$, and $d_{i \sigma}$ denotes the annihilation operator of the $3 d$ hole with the $x^{2}-y^{2}$ orbital and spin $\sigma$ at site $i$. The $w$ is a constant proportional to $\int_{0}^{\infty} r^{3} R_{3 d}(r) R_{2 p}(r) \mathrm{d} r$ where $R_{3 d}(r)$ and $R_{2 p}(r)$ are the radial wave-functions for the $3 d$ and $2 p$ states of $\mathrm{Cu}$ atom. The $D^{\alpha}(j m, \sigma)$ describes the dependence of the $E 1$ transition amplitude on the $2 p$ core-hole angular momentum and the spin of the $3 d$ hole.

In the $E 1$ transition at the $L_{2^{-}}$and $L_{3}$-edges, the initial photon having $q=q_{\mathrm{i}}, \alpha=\alpha_{\mathrm{i}}$ excites $2 p$ core hole into empty $3 d$ state, which decays back into the $2 p$ state by emitting the final photon having $q=q_{\mathrm{f}}, \alpha=\alpha_{\mathrm{f}}$. The RIXS spectra associated with this process may be expressed as

$$
\begin{aligned}
W\left(q_{\mathrm{f}} \alpha_{\mathrm{f}} ; q_{\mathrm{i}} \alpha_{\mathrm{i}}\right) & =2 \pi \sum_{f^{\prime}}\left|\sum_{n} \frac{\left\langle\Phi_{f^{\prime}}\left|H_{\mathrm{int}}\right| n\right\rangle\left\langle n\left|H_{\mathrm{int}}\right| \Phi_{\mathrm{i}}\right\rangle}{E_{\mathrm{g}}+\omega_{\mathrm{i}}-E_{n}}\right|^{2} \\
& \times \delta\left(E_{\mathrm{g}}+\omega_{\mathrm{i}}-E_{f^{\prime}}-\omega_{\mathrm{f}}\right)
\end{aligned}
$$

with $q_{\mathrm{i}} \equiv\left(\mathbf{q}_{\mathrm{i}}, \omega_{\mathrm{i}}\right), q_{\mathrm{f}} \equiv\left(\mathrm{q}_{\mathrm{f}}, \omega_{\mathrm{f}}\right),\left|\Phi_{\mathrm{i}}\right\rangle=c_{q_{\mathrm{i}} \alpha_{\mathrm{i}}}^{\dagger}|\mathrm{g}\rangle|\mathrm{vac}\rangle$, $\left|\Phi_{f^{\prime}}\right\rangle=c_{q_{\mathrm{f}} \alpha_{\mathrm{f}}}^{\dagger}\left|f^{\prime}\right\rangle \mid$ vac $\rangle$, where $|\mathrm{g}\rangle$ and $\left|f^{\prime}\right\rangle$ represent the ground state and excited states of the matter with energy $E_{\mathrm{g}}$ and $E_{f^{\prime}}$, respectively. Note that f refers to the final state of the photon and $f^{\prime}$ refers to the excited state of the electron. The $|\mathrm{vac}\rangle$ is the vacuum state for photons. The eigenstate and its energy of the intermediate state are referred to as $|n\rangle$ and $E_{n}$, respectively.

Incidentally, since the final state in the absorption coefficient $A(\omega)$ is the intermediate state in the RIXS, we have

$$
A(\omega)=2 \pi \sum_{n}\left|\left\langle n\left|H_{\mathrm{int}}\right| \Phi_{\mathrm{i}}\right\rangle\right|^{2} \delta\left(E_{\mathrm{g}}+\omega-E_{n}\right) .
$$

The explicit form is summarised in Appendix A.

\section{MAGNETIC EXCITATIONS AROUND THE CORE-HOLE SITE}

In undoped cuprates, the low-energy excitations may be well described by the two-dimensional antiferromagnetic Heisenberg Hamiltonian on a square lattice,

$$
H_{\mathrm{mag}}=J \sum_{\left\langle i, i^{\prime}\right\rangle} \mathrm{S}_{i} \cdot \mathrm{S}_{i^{\prime}}
$$

where $\mathrm{S}_{i}$ denotes the spin one half operator at site $i$, and $\left\langle i, i^{\prime}\right\rangle$ indicates that the summation runs over nearestneighbour pairs. Since our focus is not on a discussion of the magnetic dispersion, we have adopted the exchange interaction $J$ only between the nearest neighbour sites. In the thermodynamic limit, the ground state of $H_{\text {mag }}$ on a square lattice is spontaneous symmetry broken phase, that is, long-range ordering antiferromagnetic phase.

We write the ground state $|g\rangle$ of $H_{\mathrm{mag}}$ as

$$
|g\rangle=|\uparrow\rangle\left|\psi_{0}^{\uparrow}\right\rangle+|\downarrow\rangle\left|\psi_{0}^{\downarrow}\right\rangle,
$$

where $|\uparrow\rangle$ and $|\downarrow\rangle$ represent the spin states at the origin, and $\left|\psi_{0}^{\uparrow}\right\rangle$ and $\left|\psi_{0}^{\downarrow}\right\rangle$ are constructed by the bases of the rest of spins. We assume that a core hole is created at the origin as a result of absorbing photon (figure 1). In the intermediate state, the spin degrees of freedom is lost at the core-hole site, since the $3 d$ hole in the $x^{2}-y^{2}$ orbital is annihilated by the $2 p-3 d$ dipole transition. Note that the Hamiltonian in the intermediate state is similar to that for a system with a non-magnetic impurity introduced into antiferromagnet ${ }^{25,26}$.

Denoting $\left|\phi_{\eta}\right\rangle$ as the eigenstate of the intermediate Hamiltonian with eigenvalue $\epsilon_{\eta}^{\prime}$, we can express the second-order amplitude in (2.2) as

$$
\begin{aligned}
\sum_{n} \frac{H_{\mathrm{int}}|n\rangle\left\langle n\left|H_{\mathrm{int}}\right| \mathrm{g}\right\rangle}{\omega_{\mathrm{i}}+E_{\mathrm{g}}-E_{n}} & \propto \sum_{m, \sigma, \sigma^{\prime}} D^{\alpha_{\mathrm{f}}}(j m, \sigma)^{*} D^{\alpha_{\mathrm{i}}}\left(j m, \sigma^{\prime}\right) \\
& \times \sum_{\eta}|\sigma\rangle\left|\phi_{\eta}\right\rangle R\left(\epsilon_{\eta}^{\prime}\right)\left\langle\phi_{\eta} \mid \psi_{0}^{\sigma^{\prime}}\right\rangle,(3.3)
\end{aligned}
$$

with

$$
R\left(\epsilon_{\eta}^{\prime}\right)=\frac{1}{\omega_{\mathrm{i}}+\epsilon_{\mathrm{g}}-\epsilon_{\mathrm{core}}+i \Gamma-\epsilon_{\eta}^{\prime}},
$$

where $\epsilon_{\mathrm{g}}$ represents the ground state energy of $H_{\mathrm{mag}}$. The $\epsilon_{\text {core }}$ denotes the energy required to create the $3 d^{10}$ configuration and a $2 p$ core hole in the state $|j m\rangle$. The $\Gamma$ stands for the life-time broadening width of the core hole; $\Gamma \sim 0.3 \mathrm{eV}$ at the $\mathrm{Cu} L_{3}$ edge. Notice that the scattering amplitude (6) and those investigated in the remaining of this section are originated from the excitation of the single electron at the origin. A whole scattering intensity will be given by collecting up the amplitudes from all $\mathrm{Cu}$ sites.

In the scattering amplitudes leading to those excited states, we seek the invariant form with the polarization vectors $\boldsymbol{\alpha}_{\mathrm{i}}$ and $\boldsymbol{\alpha}_{\mathrm{f}}$ of the incident and scattered x-rays, spin operators $\mathrm{S}_{i}$, and the unit vector of the staggered moment $e_{m}$. To this end, it is convenient to consider a general situation that $\mathrm{e}_{\mathrm{m}}$ is pointing to an arbitrary direction, which is denoted as axis $z^{\prime}$. Then, for spin operators of the $3 d$ electron, coordinate frame of $x^{\prime}, y^{\prime}, z^{\prime}$ axes is prepared (See figure 1 (a)). On the other hand, the $3 d$ orbitals as well as spin and orbital of the core hole are described in the crystal-fixed coordinate frame with $x, y$, and $z$ axes. Since the definition of the spin coordinate system and that of the crystal-fixed system are independent, we can relate them by any method which can describe the transformation from the one to the other. We adopt here the rotation of the Euler angles $\alpha, \beta$, and 
$\gamma$ as the transformation from the crystal-fixed to the spin coordinate system 27 . Our final formulae do not depend on the specific choice of the Euler angles.

The $D^{\mu}(j m, \sigma)$ in this definition is given in table I of ${ }^{12}$. Then we introduce $P_{\sigma}^{(0)}\left(j ; \alpha_{\mathrm{f}}, \alpha_{\mathrm{i}}\right)$ and $P_{\sigma}^{(1)}\left(j ; \alpha_{\mathrm{f}}, \alpha_{\mathrm{i}}\right)$ by

$$
\begin{aligned}
\sum_{m} D^{\alpha_{\mathrm{f}}}(j m, \sigma)^{*} D^{\alpha_{\mathrm{i}}}(j m, \sigma) & \equiv P_{\sigma}^{(0)}\left(j ; \alpha_{\mathrm{f}}, \alpha_{\mathrm{i}}\right),(3.5) \\
\sum_{m} D^{\alpha_{\mathrm{f}}}(j m, \sigma)^{*} D^{\alpha_{\mathrm{i}}}(j m,-\sigma) & \equiv P_{\sigma}^{(1)}\left(j ; \alpha_{\mathrm{f}}, \alpha_{\mathrm{i}}\right),(3.6)
\end{aligned}
$$

where $-\sigma$ represent $\downarrow$ and $\uparrow$ for $\sigma=\uparrow$ and $\downarrow$, respectively. The $P_{\sigma}^{(0)}\left(j ; \alpha_{\mathrm{f}}, \alpha_{\mathrm{i}}\right)$ and $P_{\sigma}^{(1)}\left(j ; \alpha_{\mathrm{f}}, \alpha_{\mathrm{i}}\right)$ correspond to the spin-conserving and the spin-flipping processes, respectively. Polarizations of x-ray are along the $x, y$, and $z$ axes defined in the original crystal axes. Since the following analysis is confined to the $L_{3}$-edge, we fix $j=\frac{3}{2}$ and omit the argument in the expressions of $P_{\sigma}^{(0)}\left(j ; \alpha_{\mathrm{f}}, \alpha_{\mathrm{i}}\right)$ and $P_{\sigma}^{(1)}\left(j ; \alpha_{\mathrm{f}}, \alpha_{\mathrm{i}}\right)$. We list all the non-zero values of them for $j=\frac{3}{2}$ below.

$$
\begin{aligned}
& P_{\sigma}^{(0)}(x, x)=P_{\sigma}^{(0)}(y, y)=\frac{2}{15} \\
& P_{\sigma}^{(0)}(x, y)=-P_{\sigma}^{(0)}(y, x)=-\operatorname{sgn}(\sigma) \frac{\mathrm{i}}{15} \cos \beta \\
& P_{\sigma}^{(1)}(x, y)=-P_{\sigma}^{(1)}(y, x)=\frac{\mathrm{i}}{15} \exp [\mathrm{i} \gamma \operatorname{sgn}(\sigma)] \sin \beta,
\end{aligned}
$$

where $\operatorname{sgn}(\sigma)$ gives +1 and -1 for $\sigma=\uparrow$ and $\downarrow$, respectively. Note that $P^{(0)}\left(\alpha_{\mathrm{f}}, \alpha_{\mathrm{i}}\right)$ and $P_{\sigma}^{(1)}\left(\alpha_{\mathrm{f}}, \alpha_{\mathrm{i}}\right)$ are zero if $\alpha_{\mathrm{i}}=z$ and $/$ or $\alpha_{\mathrm{f}}=z$. This results from the fact that the process is restricted with the hole of the $x^{2}-y^{2}$ orbital in the ground state.

\section{A. Scattering channel with changing polarization}

As seen from (3.8) and (3.9), both $P_{\sigma}^{(0)}\left(\alpha_{\mathrm{f}}, \alpha_{\mathrm{i}}\right)$ and $P_{\sigma}^{(1)}\left(\alpha_{\mathrm{f}}, \alpha_{\mathrm{i}}\right)$ have off-diagonal elements with $\alpha_{\mathrm{i}}$ and $\alpha_{\mathrm{f}}$. This implies that the scattering channel with changing photon polarization includes both the spin-flipping and spin-conserving processes. Let us investigate them separately in the following.

\section{Spin-flipping process}

The final state arising from the spin-flipping process may be written as

$$
|F\rangle \equiv \sum_{\sigma} P_{\sigma}^{(1)}\left(\alpha_{\mathrm{f}}, \alpha_{\mathrm{i}}\right)|\sigma\rangle \sum_{\eta}\left|\phi_{\eta}\right\rangle R\left(\epsilon_{\eta}^{\prime}\right)\left\langle\phi_{\eta} \mid \psi_{0}^{-\sigma}\right\rangle .
$$

Assuming the magnetic excitation associated with the creation of core-hole at site 0 has a local character around the core-hole site, we approximate $|F\rangle$ by a linear combination of the states $\left|\psi_{1}^{( \pm)}\right\rangle=S_{0}^{ \pm}|\mathrm{g}\rangle$ and $\left|\psi_{2}^{( \pm)}\right\rangle=X^{ \pm}|\mathrm{g}\rangle$, where $\mathrm{X}=\frac{1}{z} \sum_{j} \mathrm{~S}_{j}$ with $j$ running over the nearest neighbour sites around the core-hole site. The number of the nearest neighbour sites $z$ is four and two for two and one dimensions, respectively. Spin raising and lowering operators on the core-hole site and neighbouring site are defined as $S_{0}^{ \pm}=S_{0}^{x^{\prime}} \pm \mathrm{i} S_{0}^{y^{\prime}}$ and $X^{ \pm}=X^{x^{\prime}} \pm \mathrm{i} X^{y^{\prime}}$, respectively.

Since the $\left|\psi_{n}^{( \pm)}\right\rangle$'s are not orthogonal to each other nor normalized, we need to introduce the density matrices $\left(\hat{\rho}^{( \pm)}\right)_{i, j}=\left\langle\psi_{i}^{( \pm)} \mid \psi_{j}^{( \pm)}\right\rangle$to project $|F\rangle$ onto $\left|\psi_{i}^{( \pm)}\right\rangle$'s. A procedure to determine the expansion coefficients is given in Appendix B where the projection formalism is utilised. It may seem strange the non-orthonormal set is used in the expansion. However, since the procedure described in Appendix B can determine the expansion coefficients uniquely for the finite number of the projected states, the non-orthonormal set can have a one-to-one correspondence with some orthonormal set, for instance, by means of Gram-Schmidt process. Since the physical meaning of each element of the non-orthonormal set is much clearer than that of the orthonormal one, we use the former.

Then, the final state is approximately expressed as

$$
\begin{aligned}
|F\rangle & \approx \sum_{\sigma} P_{\sigma}^{(1)}\left(\alpha_{\mathrm{f}}, \alpha_{\mathrm{i}}\right) \sum_{i j, \nu= \pm}\left|\psi_{i}^{(\nu)}\right\rangle\left(\hat{\rho}^{(\nu)-1}\right)_{i, j}\left\langle\psi_{j}^{(\nu)}\right| \\
& \times \sum_{\eta}|\sigma\rangle\left|\phi_{\eta}\right\rangle R\left(\epsilon_{\eta}^{\prime}\right)\left\langle\phi_{\eta} \mid \psi_{0}^{-\sigma}\right\rangle .
\end{aligned}
$$

This expression is rearranged as

$$
\begin{aligned}
|F\rangle & \approx P_{\downarrow}^{(1)}\left(\alpha_{\mathrm{f}}, \alpha_{\mathrm{i}}\right)\left[f_{0, \downarrow}^{(1)}\left(\omega_{i}\right) S_{0}^{-}|\mathrm{g}\rangle+\mathrm{f}_{1, \downarrow}^{(1)}\left(\omega_{\mathrm{i}}\right) \mathrm{X}^{-}|\mathrm{g}\rangle\right] \\
& +P_{\uparrow}^{(1)}\left(\alpha_{\mathrm{f}}, \alpha_{\mathrm{i}}\right)\left[f_{0, \uparrow}^{(1)}\left(\omega_{i}\right) S_{0}^{+}|\mathrm{g}\rangle+\mathrm{f}_{1, \uparrow}^{(1)}\left(\omega_{\mathrm{i}}\right) \mathrm{X}^{+}|\mathrm{g}\rangle\right] .
\end{aligned}
$$

The coefficients are given by

$$
\begin{aligned}
f_{0, \downarrow}^{(1)}\left(\omega_{i}\right) & =\sum_{j=1,2}\left(\hat{\rho}^{(-)-1}\right)_{1, j} \\
& \times\left\langle\psi_{j}^{(-)}\left|\sum_{\eta}\right| \downarrow\right\rangle\left|\phi_{\eta}\right\rangle R\left(\epsilon_{\eta}^{\prime}\right)\left\langle\phi_{\eta} \mid \psi_{0}^{\uparrow}\right\rangle, \\
f_{0, \uparrow}^{(1)}\left(\omega_{i}\right) & =\sum_{j=1,2}\left(\hat{\rho}^{(+)-1}\right)_{1, j} \\
& \times\left\langle\psi_{j}^{(+)}\left|\sum_{\eta}\right| \uparrow\right\rangle\left|\phi_{\eta}\right\rangle R\left(\epsilon_{\eta}^{\prime}\right)\left\langle\phi_{\eta} \mid \psi_{0}^{\downarrow}\right\rangle .
\end{aligned}
$$

The $f_{1, \sigma}^{(1)}\left(\omega_{i}\right)$ can be constructed from $f_{0, \sigma}^{(1)}\left(\omega_{i}\right)$ by replacing $\left(\hat{\rho}^{( \pm)-1}\right)_{1, j}$ with $\left(\hat{\rho}^{( \pm)-1}\right)_{2, j}$. Let us examine each coefficients appeared in (3.12). We suppose that the core hole site belongs to 'up spin' sublattice. This does not mean $S_{0}^{+}|g\rangle=0$ when $|g\rangle$ is the symmetry broken antiferromagnetic ground state. That is, the spin can be raised even at the 'up spin site'. Then, for example, if spin-flip excitation takes place at the core-hole site, 
two channels, from up spin to down spin and vice versa should be survived. Each cahnnel experiences different surroundings in the intermediate state through the second order process, which is materialized due to the fact that the core-hole has a finite life-time. As a result, both channels aquire different values of the coefficients. Similar explanation is also valid for the spin-flip process at the nearest neighbour sites.

In the presence of the antiferromagnetic long-range order, the coefficients $f_{0, \sigma}^{(1)}\left(\omega_{i}\right)$ and $f_{1, \sigma}^{(1)}\left(\omega_{i}\right)$ for $\sigma=\uparrow$ are expected to be different from those for $\sigma=\downarrow$. Then, let us divide them into two parts as follows.

$$
\begin{aligned}
& f_{0, \sigma}^{(1)}\left(\omega_{i}\right)=f_{0}^{(1)}\left(\omega_{i}\right)+\operatorname{sgn}(\sigma) \Delta_{\perp, 0}^{(1)}\left(\omega_{\mathrm{i}}\right), \\
& f_{1, \sigma}^{(1)}\left(\omega_{i}\right)=f_{1}^{(1)}\left(\omega_{i}\right)+\operatorname{sgn}(\sigma) \Delta_{\perp, 1}^{(1)}\left(\omega_{\mathrm{i}}\right) .
\end{aligned}
$$

It has been confirmed that $\Delta_{\perp, 0}\left(\omega_{i}\right)=\Delta_{\perp, 1}\left(\omega_{i}\right)=0$ and $f_{n, \uparrow}^{(1)}\left(\omega_{i}\right)=f_{n, \downarrow}^{(1)}\left(\omega_{i}\right)$ in the absence of the long-range order for one-dimensional system 13 . Therefore, the $\Delta_{\perp, n}^{(1)}\left(\omega_{i}\right)$ stands for the anisotropic part of the coefficient, while $f_{n}^{(1)}\left(\omega_{i}\right)$ represents the isotropic part of coefficient. Note that the anisotropic coefficient $\Delta_{\perp, 0}\left(\omega_{i}\right)$ and the coefficients for the excitations on neighbouring sites $f_{1, \sigma}^{(1)}\left(\omega_{i}\right)$ would not come out in the FCA, since the relaxation process in the intermediate state is disregarded. Inserting (3.15) and (3.16) into (3.12) with the help of (3.9), we notice that (3.12) with the Euler angles $\alpha, \beta, \gamma$ constitute an invariant form (see (3.18) in ${ }^{12}$ for isotropic terms). The result is given by

$$
\begin{aligned}
|F\rangle & \approx \frac{2 \mathrm{i}}{15}\left(\boldsymbol{\alpha}_{\mathrm{i} \perp} \times \boldsymbol{\alpha}_{\mathrm{f} \perp}\right) \cdot\left\{f_{0}^{(1)}\left(\omega_{\mathrm{i}}\right) \mathrm{S}_{0 \perp}+f_{1}^{(1)}\left(\omega_{\mathrm{i}}\right) \mathrm{X}_{\perp}\right. \\
& \left.-\mathrm{i}\left[\Delta_{\perp, 0}^{(1)}\left(\omega_{\mathrm{i}}\right) \mathrm{e}_{\mathrm{m}} \times \mathrm{S}_{0 \perp}+\Delta_{\perp, 1}^{(1)}\left(\omega_{\mathrm{i}}\right) \mathrm{e}_{\mathrm{m}} \times \mathrm{X}_{\perp}\right]\right\}|\mathrm{g}\rangle,
\end{aligned}
$$

where $\boldsymbol{\alpha}_{\mathrm{i} \perp}$ and $\boldsymbol{\alpha}_{\mathrm{f} \perp}$, respectively, are polarization vectors of the incident and scattered photon, which are projected onto the $a-b$ plane. Operators $\mathrm{S}_{0 \perp}$ and $\mathrm{X}_{\perp}$, respectively, are $\mathrm{S}_{0}$ and $\mathrm{X}$, which are projected onto the plane perpendicular to the direction of the staggered magnetic moment.

\section{Spin-conserving process}

According to (3.3), the spin-conserving process may be written as

$$
\left|F^{\prime}\right\rangle \equiv \sum_{\sigma} P_{\sigma}^{(0)}\left(\alpha_{\mathrm{f}}, \alpha_{\mathrm{i}}\right) \sum_{\eta}|\sigma\rangle\left|\phi_{\eta}\right\rangle R\left(\epsilon_{\eta}^{\prime}\right)\left\langle\phi_{\eta} \mid \psi_{0}^{\sigma}\right\rangle,
$$

where the off-diagonal elements with the polarizations are used for $P_{\sigma}^{(0)}\left(\alpha_{\mathrm{f}}, \alpha_{\mathrm{i}}\right)$. We approximate $\left|F^{\prime}\right\rangle$ by a linear combination of the states $\left|\psi_{1}\right\rangle=|\mathrm{g}\rangle,\left|\psi_{2}\right\rangle=S_{0}^{z^{\prime}}|\mathrm{g}\rangle$, and $\left|\psi_{3}\right\rangle=X^{z^{\prime}}|\mathrm{g}\rangle$. Since these states are not orthogonal to each other nor normalized, we repeat the analysis that utilises the density matrix $\hat{\rho}_{i, j} \equiv\left\langle\psi_{i} \mid \psi_{j}\right\rangle$. Hence the final state in this channel is approximately expressed as

$$
\begin{aligned}
\left|F^{\prime}\right\rangle & \approx \sum_{\sigma} P_{\sigma}^{(0)}\left(\alpha_{\mathrm{f}}, \alpha_{\mathrm{i}}\right) \sum_{i j}\left|\psi_{i}\right\rangle\left(\hat{\rho}^{-1}\right)_{i, j}\left\langle\psi_{j}\right| \\
& \times \sum_{\eta}|\sigma\rangle\left|\phi_{\eta}\right\rangle R\left(\epsilon_{\eta}^{\prime}\right)\left\langle\phi_{\eta} \mid \psi_{0}^{\sigma}\right\rangle
\end{aligned}
$$

This relation is rewritten as

$$
\left|F^{\prime}\right\rangle \approx \frac{2 \mathrm{i}}{15}\left(\boldsymbol{\alpha}_{\mathrm{i} \perp} \times \boldsymbol{\alpha}_{\mathrm{f} \perp}\right) \cdot\left[g_{0}^{(1)}\left(\omega_{\mathrm{i}}\right) \mathrm{S}_{0 \|}+g_{1}^{(1)}\left(\omega_{\mathrm{i}}\right) \mathrm{X}_{\|}\right]|\mathrm{g}\rangle
$$

where $\mathrm{S}_{0 \|}$ and $\mathrm{X}_{\|}$, respectively, represent the vector operators of $S_{0}$ and $X$ parallel to the direction of the staggered magnetic moment. Note that the amplitude associated with $\left|\psi_{1}\right\rangle$ is omitted. The definition of the expansion coefficient $g_{n}^{(1)}\left(\omega_{\mathrm{i}}\right)$ is inferred from the projection procedure in Appendix B. We have already confirmed that $g_{0}^{(1)}\left(\omega_{\mathrm{i}}\right)$ and $g_{1}^{(1)}\left(\omega_{\mathrm{i}}\right)$ were equivalent to $f_{0}^{(1)}\left(\omega_{\mathrm{i}}\right)$ and $f_{1}^{(1)}\left(\omega_{\mathrm{i}}\right)$, respectively, in the absence of long-range order ${ }^{13} .28$ Therefore, it is natural, in the presence of long-range order, to write them as

$$
\begin{aligned}
& g_{0}^{(1)}\left(\omega_{\mathrm{i}}\right)=f_{0}^{(1)}\left(\omega_{\mathrm{i}}\right)-\mathrm{i} \Delta_{\|, 0}^{(1)}\left(\omega_{\mathrm{i}}\right), \\
& g_{1}^{(1)}\left(\omega_{\mathrm{i}}\right)=f_{1}^{(1)}\left(\omega_{\mathrm{i}}\right)-\mathrm{i} \Delta_{\|, 1}^{(1)}\left(\omega_{\mathrm{i}}\right) .
\end{aligned}
$$

Here $\Delta_{\|, 0}^{(1)}\left(\omega_{\mathrm{i}}\right)$ and $\Delta_{\|, 1}^{(1)}\left(\omega_{\mathrm{i}}\right)$ correspond to the anisotropic contributions of the coefficients.

Combining the spin-conserving term (3.20) to the spinflipping term (3.17), we finally have

$$
\begin{aligned}
& |F\rangle_{1} \equiv|F\rangle+\left|F^{\prime}\right\rangle \\
& \approx \frac{2 \mathrm{i}}{15}\left(\boldsymbol{\alpha}_{\mathrm{i} \perp} \times \boldsymbol{\alpha}_{\mathrm{f} \perp}\right) \cdot\left[f_{0}^{(1)}\left(\omega_{\mathrm{i}}\right) \mathrm{S}_{0}-\mathrm{i} \Delta_{\perp, 0}^{(1)}\left(\omega_{\mathrm{i}}\right) \mathrm{e}_{\mathrm{m}} \times \mathrm{S}_{0}\right. \\
& \left.\quad-\mathrm{i} \Delta_{\|, 0}^{(1)}\left(\omega_{\mathrm{i}}\right) \mathrm{e}_{\mathrm{m}}\left(\mathrm{e}_{\mathrm{m}} \cdot \mathrm{S}_{0}\right)\right]|\mathrm{g}\rangle \\
& +\frac{2 \mathrm{i}}{15}\left(\boldsymbol{\alpha}_{\mathrm{i} \perp} \times \boldsymbol{\alpha}_{\mathrm{f} \perp}\right) \cdot\left[f_{1}^{(1)}\left(\omega_{\mathrm{i}}\right) \mathrm{X}-\mathrm{i} \Delta_{\perp, 1}^{(1)}\left(\omega_{\mathrm{i}}\right) \mathrm{e}_{\mathrm{m}} \times \mathrm{X}\right. \\
& \left.\quad-\mathrm{i} \Delta_{\|, 1}^{(1)}\left(\omega_{\mathrm{i}}\right) \mathrm{e}_{\mathrm{m}}\left(\mathrm{e}_{\mathrm{m}} \cdot \mathrm{X}\right)\right]|\mathrm{g}\rangle .
\end{aligned}
$$

The terms containing $e_{m}$ represent the effect of the long range order, that is, that of the broken symmetry in spin space. If $e_{m}$ is defined on the A sublattice and the same $\mathrm{e}_{\mathrm{m}}$ is used on the B sublattice, $\Delta_{\|, 0}^{(1)}\left(\omega_{\mathrm{i}}\right)$ and $\Delta_{\|, 1}^{(1)}\left(\omega_{\mathrm{i}}\right)$, respectively, take the same value in both sublattices. On the other hand, the values of $\Delta_{\perp, 0}^{(1)}\left(\omega_{\mathrm{i}}\right)$ and $\Delta_{\perp, 1}^{(1)}\left(\omega_{\mathrm{i}}\right)$ in sublattice $B$, respectively, are obtained by changing entire sign of those in sublattice A, respectively. 


\section{B. Scattering channel without changing polarization}

Since only $P_{\sigma}^{(0)}\left(\alpha_{\mathrm{f}}, \alpha_{\mathrm{i}}\right)$ has the non-zero diagonal elements with $\alpha_{\mathrm{i}}$ and $\alpha_{\mathrm{f}}$, (3.3) may be expressed as

$$
|F\rangle_{2} \equiv \sum_{\sigma} P_{\sigma}^{(0)}(\alpha, \alpha)|\sigma\rangle \sum_{\eta}\left|\phi_{\eta}\right\rangle R\left(\epsilon_{\eta}^{\prime}\right)\left\langle\phi_{\eta} \mid \psi_{0}^{\sigma}\right\rangle
$$

We see that the FCA could not give rise to spin excitations in this process because the diagonal element $P_{\sigma}^{(0)}(\alpha, \alpha)$ is independent of $\sigma$. Since the total spin is conserved, $|F\rangle_{2}$ may be expressed by $|g\rangle, S_{0}^{z^{\prime}}|\mathrm{g}\rangle, X^{z^{\prime}}|\mathrm{g}\rangle$, $S_{0}^{z^{\prime}} X^{z^{\prime}}|\mathrm{g}\rangle$, and $\frac{1}{2}\left(S_{0}^{+} X^{-}+S_{0}^{-} X^{+}\right)|\mathrm{g}\rangle$. Similar to the procedure resorted in the preceding subsection, $|F\rangle_{2}$ is approximated by a linear combination of these states with the help of the density matrix. Hence $|F\rangle_{2}$ is approximately expressed as

$$
\begin{aligned}
|F\rangle_{2} & \approx \frac{2}{15}\left(\boldsymbol{\alpha}_{\mathrm{i} \perp} \cdot \boldsymbol{\alpha}_{\mathrm{f} \perp}\right) f_{2}^{(2)}\left(\omega_{\mathrm{i}}\right) \mathrm{S}_{0} \cdot \mathrm{X}|\mathrm{g}\rangle \\
& +\frac{2}{15}\left(\boldsymbol{\alpha}_{\mathrm{i} \perp} \cdot \boldsymbol{\alpha}_{\mathrm{f} \perp}\right)\left[\Lambda^{(2)}\left(\omega_{\mathrm{i}}\right)\left(\mathrm{e}_{\mathrm{m}} \cdot \mathrm{S}_{0}\right)\left(\mathrm{e}_{\mathrm{m}} \cdot \mathrm{X}\right)\right. \\
& \left.+\Delta_{\|, 0}^{(2)}\left(\omega_{\mathrm{i}}\right)\left(\mathrm{e}_{\mathrm{m}} \cdot \mathrm{S}_{0}\right)+\Delta_{\|, 1}^{(2)}\left(\omega_{\mathrm{i}}\right)\left(\mathrm{e}_{\mathrm{m}} \cdot \mathrm{X}\right)\right] \mid \mathrm{g}(3.25)
\end{aligned}
$$

where the amplitude associated with $|\mathrm{g}\rangle$ is omitted. The terms containing $\mathrm{e}_{\mathrm{m}}$ represent the effect of broken symmetry in spin space. The expansion coefficients for $S_{0}^{z^{\prime}}|g\rangle$ and $X^{z^{\prime}}|\mathrm{g}\rangle$ are denoted as $\Delta_{\|, 0}^{(2)}\left(\omega_{\mathrm{i}}\right)$ and $\Delta_{\|, 1}^{(2)}\left(\omega_{\mathrm{i}}\right)$, respectively, while those defined for $S_{0}^{z^{\prime}} X^{z^{\prime}}|\mathrm{g}\rangle$ and $\frac{1}{2}\left(S_{0}^{+} X^{-}+\right.$ $\left.S_{0}^{-} X^{+}\right)|\mathrm{g}\rangle$ are divided into the isotropic term $f_{2}^{(2)}\left(\omega_{\mathrm{i}}\right)$ and anisotropic term $\Lambda^{(2)}\left(\omega_{\mathrm{i}}\right)$. If $\mathrm{e}_{\mathrm{m}}$ is defined on the $\mathrm{A}$ sublattice and the same $e_{m}$ is used on the B sublattice, $\Lambda^{(2)}\left(\omega_{\mathrm{i}}\right)$ take the same value in both sublattices. On the other hand, the values of $\Delta_{\|, 0}^{(2)}\left(\omega_{\mathrm{i}}\right)$ and $\Delta_{\|, 1}^{(2)}\left(\omega_{\mathrm{i}}\right)$ in sublattice $\mathrm{B}$, respectively, are obtained by changing entire sign of those in sublattice A, respectively.

\section{EVALUATION OF THE COEFFICIENTS}

Various coefficients defined in the preceding section could be evaluated by diagonalizing the Heisenberg Hamiltonian on finite-size clusters. Since the excitations are localized around the core-hole site, the calculation on small clusters may give reliable estimates to the coefficients. We consider a cluster of 13 spins shown in figure 2. A complication is that analysis on finite-size cluster cannot provide spontaneous symmetry breaking ground state. In order to break the spherical symmetry in spin space, we assume that the spins on the boundary are subjected to the molecular field, $-J\left|\left\langle S_{0}^{z^{\prime}}\right\rangle\right|$, per bond. The expectation value of $S_{0}^{z^{\prime}}$ is determined self-consistently as $\left\langle S_{0}^{z^{\prime}}\right\rangle=0.394$. The coefficients in the RIXS process are evaluated by diagonalizing the Hamiltonian matrices

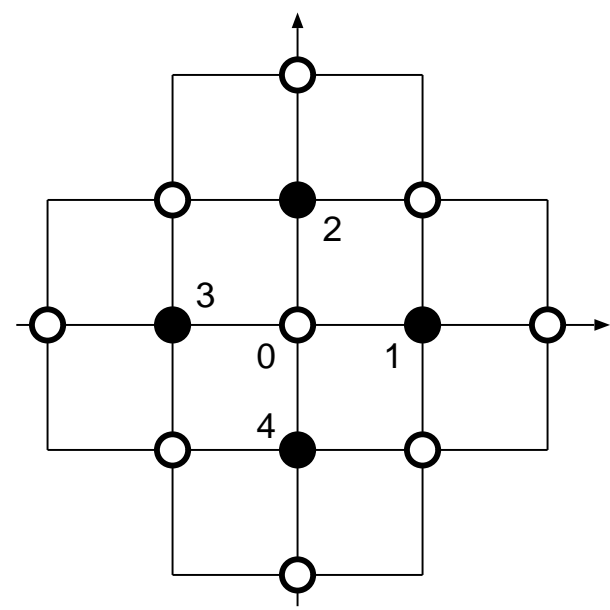

FIG. 2: A cluster of 13 spins used to evaluate the coefficients. The spin at site 0 is annihilated in the intermediate state. Spins on the boundary are subjected to the molecular field from spins outside the cluster.

in the ground state and in the intermediate state. Table \shows the calculated results at $\omega_{i}$ giving the maximum absorption coefficient with $\Gamma / J=2.4$ and 1.0. The values for $\Gamma / J=2.4$ may correspond to $\mathrm{La}_{2} \mathrm{CuO}_{4}$ and $\mathrm{Sr}_{2} \mathrm{CuO}_{2} \mathrm{Cl}_{2}$. Note that the coefficients have dimensions of (energy) ${ }^{-1}$ as seen from right-hand side of (3.3). The coefficients not shown there are small, and will be neglected in the calculation of the RIXS spectra in the next section.

As seen from (3.23) and (3.25), it is obvious qualitatively that the origin of the anisotropic terms, which include the unit vector representing the staggered moment $\left(e_{m}\right)$, is attributed to the broken symmetry of the ground state in spin space. In quantitative sense, the magnitudes of such terms are expected to develop as increasing the staggered moment. This is confirmed in Appendix C for a finite-size ring of spins.

\section{ANALYSIS OF RIXS SPECTRA FROM UNDOPED CUPRATES}

Now, we are in a position to calculate the RIXS spectra. It is preferable to treating a larger system since the spin excitations propagate through the entire crystal in the final state. Thus, we employ the results of the $1 / S$ expansion to the spin operators, which practically corresponds to taking into account of an infinite system effect as well as the interaction among the magnetic excitations. In doing so, we proceed the analysis by dividing the RIXS spectra into two channels, with and without changing photon polarization. 
TABLE I: Various coefficients in units of $1 / J$ in the two-dimensional cluster of 13 spins: (a) coefficients for isotropic terms and (b) coefficients for anisotropic terms. The incident photon energy $\omega_{i}$ is set to give the maximum absorption coefficient.

\begin{tabular}{ccccc}
\hline \hline & \multicolumn{2}{c}{ (a) Isotropic coefficients } & \multicolumn{2}{c}{ (b) Anisotropic coefficients } \\
\hline$\Gamma / J$ & $f_{0}^{(1)}\left(\omega_{\mathrm{i}}\right)$ & $f_{2}^{(2)}\left(\omega_{\mathrm{i}}\right)$ & $\Delta_{\perp, 0}^{(1)}\left(\omega_{\mathrm{i}}\right)$ & $\Lambda^{(2)}\left(\omega_{\mathrm{i}}\right)$ \\
\hline 2.4 & $(-0.087,-0.367)$ & $(0.177,-0.320)$ & $(-0.093,0.048)$ & $(0.071,-0.002)$ \\
1.0 & $(-0.263,-0.656)$ & $(0.082,-0.891)$ & $(-0.209,0.334)$ & $(0.182,-0.207)$ \\
\hline \hline
\end{tabular}

\section{A. Scattering channel with changing polarization}

Since $\boldsymbol{\alpha}_{\mathrm{f} \perp}$ and $\boldsymbol{\alpha}_{\mathrm{i} \perp}$ are polarization vectors projected onto the $a$ - $b$ plane, $\boldsymbol{\alpha}_{\mathrm{f} \perp} \times \boldsymbol{\alpha}_{\mathrm{i} \perp}$ is parallel to the $c$ axis. In undoped cuprates such as $\mathrm{La}_{2} \mathrm{CuO}_{4}$ and $\mathrm{Sr}_{2} \mathrm{CuO}_{2} \mathrm{Cl}_{2}$, the staggered magnetization aligns along the $(1,1,0)$ direction in the $\mathrm{CuO}_{2}$ plane ${ }^{29}$. Therefore the anisotropic terms proportional to $\mathrm{e}_{\mathrm{m}}$ could not come out. We collect up the remaining amplitudes from all $\mathrm{Cu}$ sites, where (3.23) is multiplied by the weight $\exp \left(\mathrm{iq} \cdot \mathrm{r}_{\mathrm{i}}\right)$ at the corehole site $\mathrm{r}_{i}$ with momentum transfer $\mathrm{q} \equiv \mathrm{q}_{\mathrm{i}}-\mathrm{q}_{\mathrm{f}}$. Thereby we obtain

$$
\begin{aligned}
& W\left(q_{\mathrm{f}}, \boldsymbol{\alpha}_{\mathrm{f}} ; q_{\mathrm{i}}, \boldsymbol{\alpha}_{\mathrm{i}}\right) \\
& =\frac{w^{4}}{4 \omega_{\mathrm{i}} \omega_{\mathrm{f}}}\left(\frac{2}{15}\right)^{2}\left(\boldsymbol{\alpha}_{\mathrm{i} \perp} \times \boldsymbol{\alpha}_{\mathrm{f} \perp}\right)^{2} Y^{(1)}\left(\omega_{\mathrm{i}} ; \mathrm{q}, \omega\right) .
\end{aligned}
$$

with $\omega \equiv \omega_{\mathrm{i}}-\omega_{\mathrm{f}}$ is defined by

$$
Y^{(1)}\left(\omega_{\mathrm{i}} ; \mathrm{q}, \omega\right)=\int\left\langle Z^{(1) \dagger}\left(\omega_{\mathrm{i}} ; \mathrm{q}, t\right) Z^{(1)}\left(\omega_{\mathrm{i}} ; \mathrm{q}, 0\right)\right\rangle \mathrm{e}^{\mathrm{i} \omega \mathrm{t}} \mathrm{d} t
$$

where

$$
\begin{aligned}
Z^{(1)}\left(\omega_{\mathrm{i}} ; \mathrm{q}\right) & =f_{0}^{(1)}\left(\omega_{\mathrm{i}}\right)\left[S_{a}^{x^{\prime}}(-\mathrm{q})+S_{b}^{x^{\prime}}(-\mathrm{q})\right] \\
& +\mathrm{i} \Delta_{\perp, 0}^{(1)}\left(\omega_{\mathrm{i}}\right)\left[S_{a}^{y^{\prime}}(-\mathrm{q})-S_{b}^{y^{\prime}}(-\mathrm{q})\right] .
\end{aligned}
$$

Here the time dependent operator of an arbitrary operator $A$ is defined as $A(t)=\mathrm{e}^{i H_{\operatorname{mag}} t} A \mathrm{e}^{-i H_{\text {mag }} t}$. The Fourier transforms of the spin operators are given by

$$
\begin{aligned}
& \mathrm{S}_{a}(-\mathrm{q})=(2 / N)^{\frac{1}{2}} \sum_{i \in \mathrm{A}} \mathrm{S}_{i} \exp \left(\mathrm{iq} \cdot \mathrm{r}_{\mathrm{i}}\right), \\
& \mathrm{S}_{b}(-\mathrm{q})=(2 / N)^{\frac{1}{2}} \sum_{i \in \mathrm{B}} \mathrm{S}_{i} \exp \left(\mathrm{iq} \cdot \mathrm{r}_{\mathrm{i}}\right),
\end{aligned}
$$

where the sum is taken over site $i$ on the A or B sublattices. The $x^{\prime}, y^{\prime}$, and $z^{\prime}$ axes are defined as directing to $(0,0,1),(1,-1,0)$, and $(1,1,0)$, respectively. The spinflip excitations on the neighbouring sites to the core hole are neglected, because their amplitudes are quite small.

We expand the spin operators by means of magnon operators in the $1 / S$-expansion method, which is briefly summarised in Appendix D. In their expressions, momenta are defined within the first magnetic Brillouin zone (MBZ). When momentum q lies outside the first MBZ, $\mathrm{S}_{a}(-\mathrm{q})$ and $\mathrm{S}_{b}(-\mathrm{q})$ are replaced by $\mathrm{S}_{a}([-\mathrm{q}])$ and $\operatorname{sgn}\left(\gamma_{G}\right) S_{b}([-q])$, respectively, where $q$ is put back into the first $\mathrm{MBZ}$ by a reciprocal lattice vector $\mathrm{G}$. That is,
$\mathrm{q}=[\mathrm{q}]+\mathrm{G}$ with $[\mathrm{q}]$ lying inside the first MBZ. The $\operatorname{sgn}\left(\gamma_{\mathrm{k}}\right)$ denotes the sign of $\gamma_{\mathrm{k}}$, where $\gamma_{\mathrm{k}}=\frac{1}{2}\left(\cos k_{x}+\right.$ $\left.\cos k_{y}\right)$ with $\mathrm{k}$ in units of $1 / a$ ( $a$ is the lattice constant). For example, $\gamma_{\mathrm{G}}=-1$ for $\mathrm{G}=(\pi, \pi)$. With these notations together with the magnon operators $\alpha_{[-\mathrm{q}]}^{\dagger}$ and $\beta_{[-\mathrm{q}]}^{\dagger}, Z^{(1)}\left(\omega_{\mathrm{i}} ; \mathrm{q}\right)$ is expressed as

$Z^{(1)}\left(\omega_{\mathrm{i}} ; \mathrm{q}\right)=\sqrt{2 S} M\left(\omega_{\mathrm{i}} ; \mathrm{q}\right)\left(\alpha_{[-\mathrm{q}]}^{\dagger}+\operatorname{sgn}\left(\gamma_{\mathrm{G}}\right) \beta_{[-\mathrm{q}]}^{\dagger}\right)+\cdots$,

where

$$
\begin{aligned}
M\left(\omega_{\mathrm{i}} ; \mathrm{q}\right) \equiv & \frac{\ell_{[\mathrm{q}]}}{2}\left\{f_{0}^{(1)}\left(\omega_{\mathrm{i}}\right)\left[1-\operatorname{sgn}\left(\gamma_{\mathrm{G}}\right) x_{[\mathrm{q}]}\right]\right. \\
& \left.+\mathrm{i} \Delta_{\perp, 0}^{(1)}\left(\omega_{\mathrm{i}}\right)\left[1+\operatorname{sgn}\left(\gamma_{\mathrm{G}}\right) x_{[\mathrm{q}]}\right]\right\} .
\end{aligned}
$$

The definitions of $\ell_{\mathrm{q}}$ and $x_{\mathrm{q}}$ are found in Appendix D [(D10)] and use has been made of the relations $\ell_{-\mathrm{q}}=\ell_{\mathrm{q}}$ and $x_{-\mathrm{q}}=x_{\mathrm{q}}$. Therefore $Y^{(1)}\left(\omega_{\mathrm{i}} ; \mathrm{q}, \omega\right)$ consists of the $\delta$-function peak, which is located at

$$
\omega=J S z\left(1+\frac{A}{2 S}\right) \epsilon_{[\mathrm{q}]},
$$

where $A=0.1579$ is the first order correction in the $1 / S$ expansion (see Appendix D ${ }^{30}$.

Figure 3 shows $Y^{(1)}\left(\omega_{\mathrm{i}} ; \mathrm{q}, \omega\right)$ as a function of $\omega$ for $\mathrm{q}$ along the symmetry directions with $\Gamma / J=2.4$. A notable aspect is that the intensities diverge at $\mathrm{q}=(0,0)$ and $(\pi, \pi)$. The corresponding integrated intensity is given by

$$
I^{(1)}\left(\omega_{\mathrm{i}} ; \mathrm{q}\right) \equiv \int Y^{(1)}\left(\omega_{\mathrm{i}} ; \mathrm{q}, \omega\right) \frac{\mathrm{d} \omega}{2 \pi}=2(2 S)\left|M\left(\omega_{\mathrm{i}} ; \mathrm{q}\right)\right|^{2} .
$$

Figure 4 shows $I^{(1)}\left(\omega_{\mathrm{i}} ; \mathrm{q}\right)$ for q along symmetry directions with $\Gamma / J=2.4$. The intensities are enhanced around $q=$ $(0,0)$ as $1 /|q|$. Since the contribution from the isotropic term vanishes around $\mathrm{q} \sim 0$, the enhancement is due to the finite value of the anisotropic coefficient $\Delta_{\perp, 0}^{(1)}\left(\omega_{\mathrm{i}}\right)$. On the other hand, the divergence around $\mathrm{q}=(\pi, \pi)$ is brought about by the isotropic term, which is why the behaviour is irrelevant of presence of the anisotropic term.

It has been observed in the RIXS experiments ${ }^{2}-4$ that the intensity of magnon peak increases significantly with $q \rightarrow 0$. Such increase is consistent with the effects of the anisotropic terms. So far, the increase of intensity has 

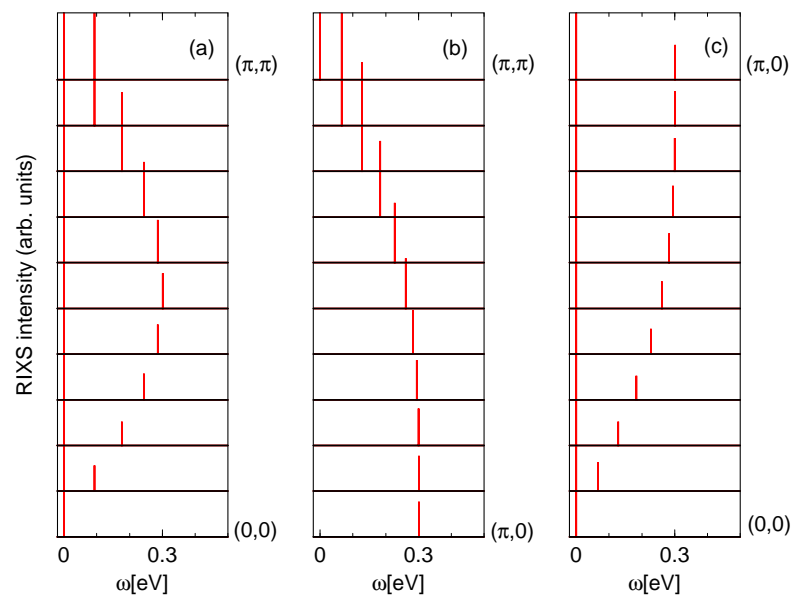

FIG. 3: $Y^{(1)}\left(\omega_{\mathrm{i}} ; \mathrm{q}, \omega\right)$ as a function of $\omega$ for q along symmetry directions. The $\omega_{\mathrm{i}}$ is set to give rise to the peak in the absorption spectra. $J=130 \mathrm{meV}$ and $\Gamma / J=2.4$. The intensities diverge at $\mathrm{q}=0$ and $(\pi, \pi)$.

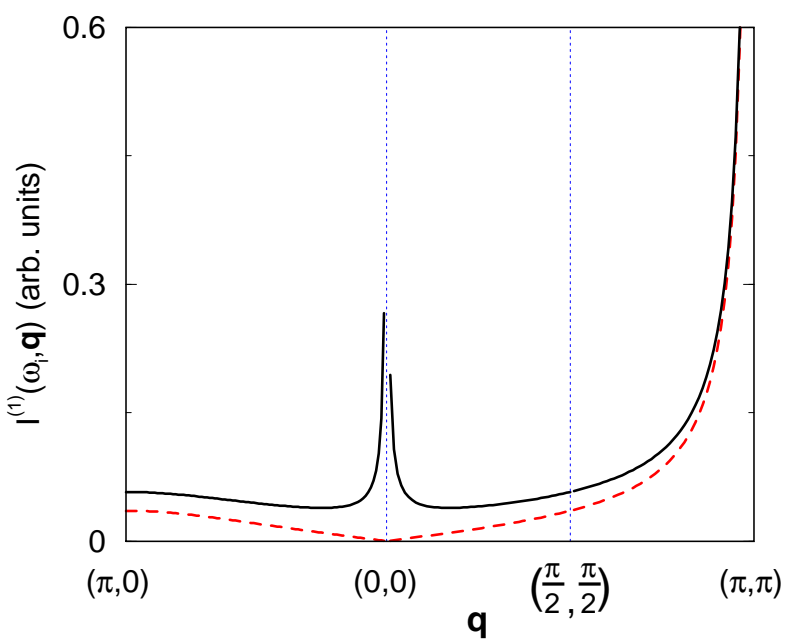

FIG. 4: Intensity $I^{(1)}\left(\omega_{\mathrm{i}} ; \mathrm{q}\right)$ of the $\delta$-function peak arising from the one-magnon excitation as a function of q along symmetry directions. The $\omega_{\mathrm{i}}$ is the photon energy giving rise to the peak in the absorption spectra. $\Gamma / J=2.4$. The (black) solid and (red) broken lines are the results with and without including $\Delta_{\perp, 0}^{(1)}\left(\omega_{\mathrm{i}}\right)$, respectively.

been interpreted simply as the contribution from elastic scattering. To confirm the effects of anisotropic terms, it may be necessary to examine carefully the spectra with subtracting systematically the contribution of elastic scattering around $\mathrm{q} \sim 0$.

It should be noted here that there exists non-linear terms which make the one-magnon excitation split into three-magnon excitations in the second order correction of the $1 / S$-expansion ${ }^{12,31}$. Accordingly $Y^{(1)}\left(\omega_{i} ; \mathrm{q}, \omega\right)$ contains the energy continuum of the three-magnon excitations in addition to the $\delta$-function peak mentioned above. The contribution from the three-magnon excitation grows gradually when $q$ is near the boundary of the first MBZ. See figure 7 in 12 for such RIXS spectra.

\section{B. Scattering channel without changing polarization}

In order to calculate the RIXS intensity in this channel, we collect up the amplitudes from all the $\mathrm{Cu}$ sites with the use of (3.25). We obtain

$$
\begin{aligned}
& W\left(q_{\mathrm{f}}, \boldsymbol{\alpha}_{\mathrm{f}} ; q_{\mathrm{i}}, \boldsymbol{\alpha}_{\mathrm{i}}\right) \\
= & \frac{w^{4}}{4 \omega_{\mathrm{i}} \omega_{\mathrm{f}}}\left(\frac{2}{15}\right)^{2}\left(\boldsymbol{\alpha}_{\mathrm{i} \perp} \cdot \boldsymbol{\alpha}_{\mathrm{f} \perp}\right)^{2} Y^{(2)}\left(\omega_{\mathrm{i}} ; \mathrm{q}, \omega\right) .
\end{aligned}
$$

The correlation function $Y^{(2)}\left(\omega_{\mathrm{i}} ; \mathrm{q}, \omega\right)$ is defined by

$$
Y^{(2)}\left(\omega_{\mathrm{i}} ; \mathrm{q}, \omega\right)=\int\left\langle Z^{(2) \dagger}\left(\omega_{\mathrm{i}} ; \mathrm{q}, t\right) Z^{(2)}\left(\omega_{\mathrm{i}} ; \mathrm{q}, 0\right)\right\rangle \mathrm{e}^{\mathrm{i} \omega \mathrm{t}} \mathrm{d} t,
$$

where

$$
\begin{aligned}
Z^{(2)}\left(\omega_{\mathrm{i}} ; \mathrm{q}\right) & =f_{2}^{(2)}\left(\omega_{\mathrm{i}}\right)\left[(\mathrm{S} \cdot \mathrm{X})_{a}(-\mathrm{q})+(\mathrm{S} \cdot \mathrm{X})_{b}(-\mathrm{q})\right] \\
& +\Lambda^{(2)}\left(\omega_{\mathrm{i}}\right)\left[\left(S^{z^{\prime}} X^{z^{\prime}}\right)_{a}(-\mathrm{q})+\left(S^{z^{\prime}} X^{z^{\prime}}\right)_{b}(-\mathrm{q})\right] \\
& +\cdots
\end{aligned}
$$

The Fourier transform $\left(S^{\mu^{\prime}} X^{\mu^{\prime}}\right)_{a}(-\mathrm{q})$ and $\left(S^{\mu^{\prime}} X^{\mu^{\prime}}\right)_{b}(-\mathrm{q})$ for $\mu^{\prime}=x^{\prime}, y^{\prime}$, and $z^{\prime}$ are introduced as follows.

$$
\begin{aligned}
& \left(S^{\mu^{\prime}} X^{\mu^{\prime}}\right)_{a}(-\mathrm{q})=(2 / N)^{\frac{1}{2}} \sum_{i \in \mathrm{A}} S_{i}^{\mu^{\prime}} \frac{1}{4} \sum_{\delta} S_{i+\delta}^{\mu^{\prime}} \mathrm{e}^{\mathrm{i} q \cdot r_{\mathrm{i}}} \\
& =(2 / N)^{\frac{1}{2}} \sum_{\mathrm{k}} S_{a}^{\mu^{\prime}}(\mathrm{k}) S_{b}^{\mu^{\prime}}(-[\mathrm{k}+\mathrm{q}]) \gamma_{[\mathrm{k}+\mathrm{q}]}, \\
& \left(S^{\mu^{\prime}} X^{\mu^{\prime}}\right)_{b}(-\mathrm{q})=(2 / N)^{\frac{1}{2}} \sum_{i \in \mathrm{B}} S_{i}^{\mu^{\prime}} \frac{1}{4} \sum_{\delta} S_{i+\delta}^{\mu^{\prime}} \mathrm{e}^{\mathrm{i} \mathrm{q} \cdot \mathrm{r}_{\mathrm{i}}} \\
& =(2 / N)^{\frac{1}{2}} \sum_{\mathrm{k}} S_{b}^{\mu^{\prime}}(\mathrm{k}) S_{a}^{\mu^{\prime}}(-[\mathrm{k}+\mathrm{q}]) \gamma_{[\mathrm{k}+\mathrm{q}]} \operatorname{sgn}\left(\gamma_{\mathrm{G}}\right) .
\end{aligned}
$$

Here the sum over $\delta$ is carried out on the nearest neighbour sites around site $i$.

Expanding $Z^{(2)}\left(\omega_{i} ; q\right)$ in terms of magnon operators within the $1 / S$-expansion(see Appendix (D), we obtain

$$
Z^{(2)}\left(\omega_{\mathrm{i}} ; \mathrm{q}\right)=(2 S) \sum_{\mathrm{k}} N\left(\omega_{\mathrm{i}} ; \mathrm{q}, \mathrm{k}\right) \alpha_{-[\mathrm{q}+\mathrm{k}]}^{\dagger} \beta_{\mathrm{k}}^{\dagger}+\cdots
$$

with $\mathrm{k}$ running within the first MBZ, and

$$
\begin{aligned}
& N\left(\omega_{\mathrm{i}} ; \mathrm{q}, \mathrm{k}\right) \\
= & f_{2}^{(2)}\left(\omega_{\mathrm{i}}\right) \frac{\ell_{[\mathrm{q}+\mathrm{k}]} \ell_{\mathrm{k}}}{2}\left\{-\left(1+\gamma_{\mathrm{q}}\right)\left[x_{\mathrm{k}}+\operatorname{sgn}\left(\gamma_{\mathrm{G}}\right) x_{[\mathrm{q}+\mathrm{k}]}\right]\right. \\
& \left.+\left[\gamma_{\mathrm{k}}+\operatorname{sgn}\left(\gamma_{\mathrm{G}}\right) \gamma_{[\mathrm{q}+\mathrm{k}]}\right]\left[1+\operatorname{sgn}\left(\gamma_{\mathrm{G}}\right) x_{[\mathrm{q}+\mathrm{k}]} x_{\mathrm{k}}\right]\right\} \\
- & \Lambda^{(2)}\left(\omega_{\mathrm{i}}\right) \frac{\ell_{[\mathrm{q}+\mathrm{k}]} \ell_{\mathrm{k}}}{2}\left(1+\gamma_{\mathrm{q}}\right)\left[x_{\mathrm{k}}+\operatorname{sgn}\left(\gamma_{\mathrm{G}}\right) x_{[\mathrm{q}+\mathrm{k}]}\right](5.16)
\end{aligned}
$$



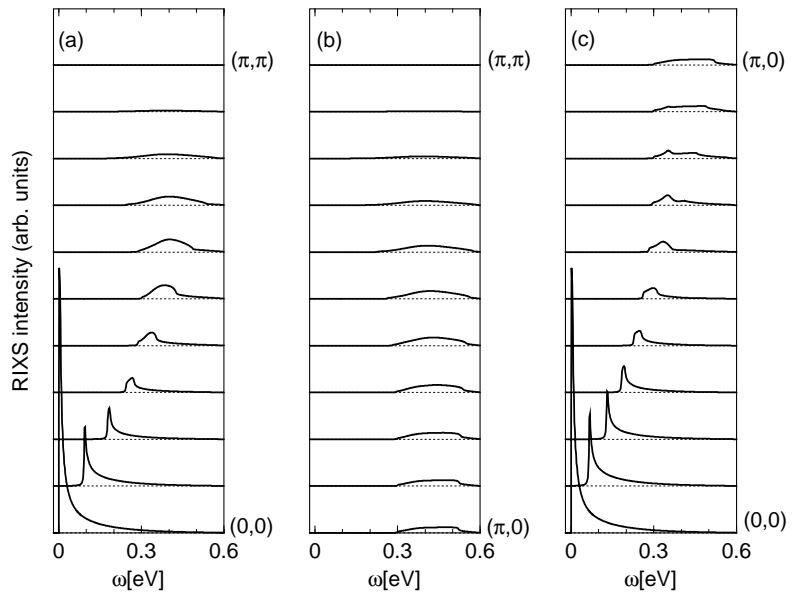

FIG. 5: $Y^{(2)}\left(\omega_{\mathrm{i}} ; \mathrm{q}, \omega\right)$ as a function of $\omega$ for $\mathrm{q}$ along symmetry directions. The $\omega_{\mathrm{i}}$ is set to give rise to the peak in the absorption spectra. $J=130 \mathrm{meV}$ and $\Gamma / J=2.4$.

This expression is valid even when $q$ is outside of the first MBZ. Note that when $\Lambda^{(2)}\left(\omega_{\mathrm{i}}\right)=0, N\left(\omega_{\mathrm{i}} ; \mathrm{q}, \mathrm{k}\right)$ vanishes at $\mathrm{q}=(0,0)$ and $(\pi, \pi)^{32}$. Note also that the isotropic terms of the two-magnon part are the same as those obtained for the $K$-edge RIXS, where no anisotropic term exists $32-34$.

From (5.15), we see that $Y^{(2)}\left(\omega_{i} ; \mathrm{q}, \omega\right)$ consists of the energy continuum of the two-magnon excitations. Since two magnons are created at neighbouring sites through x-ray scattering, inclusion of the magnon-magnon interaction is crucial to obtain the spectral shape. As already discussed in 32 , the magnon-magnon interaction in the $1 / S$-expansion could be divided into a separable form so that the $t$-matrix of the scattering is neatly evaluated. We resort to the similar evaluation. Figure 5 shows $Y^{(2)}\left(\omega_{\mathrm{i}} ; \mathrm{q}, \omega\right)$ as a function of $\omega$ for q along the symmetry directions. We find rapid enhancement of the intensity is brought about by the presence of the anisotropic terms as $|\mathrm{q}|$ goes to $(0,0)$. Without them, in contrast, the intensity diminishes in this limit as shown in figure 8 of 12 . We see the peak energy decreases with $|\mathrm{q}|$ approaching zero. At $\mathrm{q}=0$, the peak energy becomes very close to zero, $\sim 0.025 \mathrm{eV}$. It may be a difficult task to distinguish the spectral peak from the elastic peak. However a careful study on the q-dependence of the spectra may clarify such effect of the anisotropic terms.

The frequency integrated intensity $I^{(2)}\left(\omega_{\mathrm{i}} ; \mathrm{q}\right)$ may be given by

$$
\begin{aligned}
I^{(2)}\left(\omega_{\mathrm{i}} ; \mathrm{q}\right) & \equiv \int Y^{(2)}\left(\omega_{\mathrm{i}} ; \mathrm{q}, \omega\right) \frac{\mathrm{d} \omega}{2 \pi} \\
& =(2 S)^{2} \sum_{\mathrm{k}}\left|N\left(\omega_{\mathrm{i}} ; \mathrm{q}, \mathrm{k}\right)\right|^{2} .
\end{aligned}
$$

Figure [6 shows $I^{(2)}\left(\omega_{\mathrm{i}} ; \mathrm{q}\right)$ for q along symmetry directions. Notice that $I^{(2)}\left(\omega_{\mathrm{i}} ; \mathrm{q}\right)$ diverges logarithmically when $|\mathrm{q}|$ approaches zero. It demonstrates a possibility that this logarithmic enhancement can be recognized

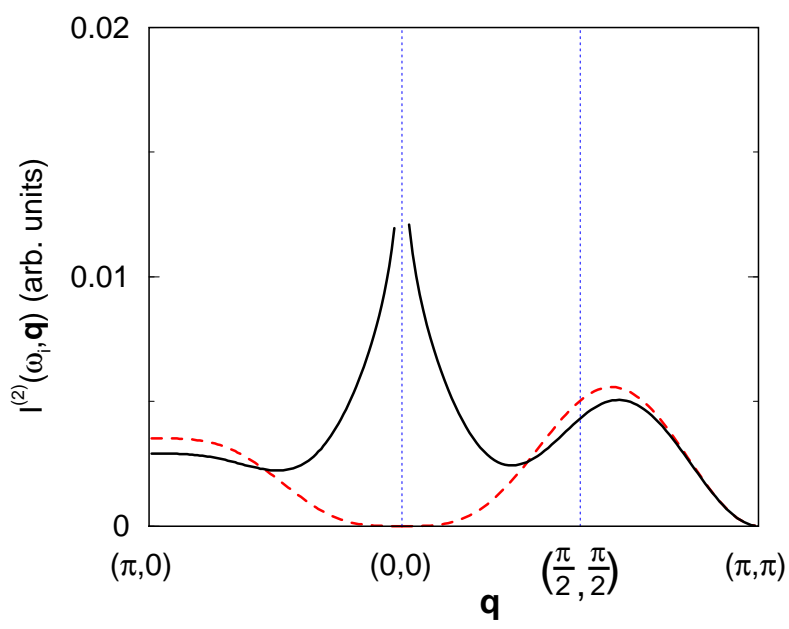

FIG. 6: Frequency-integrated intensity $I^{(2)}\left(\omega_{\mathrm{i}} ; \mathrm{q}\right)$ of the continuous spectra arising from two-magnon excitations as a function of $\mathrm{q}$ along symmetry directions. The $\omega_{\mathrm{i}}$ is the photon energy giving rise to the peak in the absorption spectra. $\Gamma / J=2.4$. The (black) solid and (red) broken lines represent the results obtained with and without the anisotropic term, respectively.

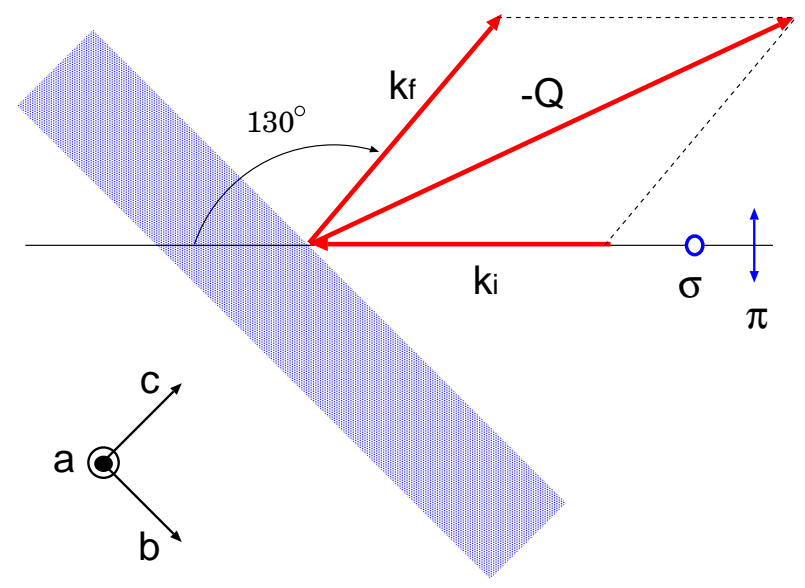

FIG. 7: Schematic view of the scattering geometry. The angle between the incident and scattered x-rays is fixed at 130 degrees. The scattering plane contains the $c$ axis.

at a region where $\mathrm{q}$ is away from $(0,0)$.

\section{Polarization dependence}

We consider a scattering geometry used in the experiments of $\mathrm{La}_{2} \mathrm{CuO}_{4} \frac{3}{3}$ and $\mathrm{Sr}_{2} \mathrm{CuO}_{2} \mathrm{Cl}_{2} \frac{4}{2}$. It is schematically shown in figure 7 for $\mathrm{q}$ along $(0,0)-(0, \pi)$ direction, where the angle between the incident and the scattered $\mathrm{x}$ ray is fixed at 130 degrees. The scattering plane includes the $b(x)$ and $c(z)$ axes.

Then $\boldsymbol{\alpha}_{\mathrm{i} \perp}=(1,0,0)$ for the $\sigma$ polarization and $\boldsymbol{\alpha}_{\mathrm{i} \perp}=$ $\left(0, \alpha_{\mathrm{i}}^{\pi}, 0\right)$ for the $\pi$ polarization in the incident x-ray, while $\boldsymbol{\alpha}_{\mathrm{f} \perp}=(1,0,0)$ for the $\sigma^{\prime}$ polarization and $\boldsymbol{\alpha}_{\mathrm{f} \perp}=$ 
$\left(0, \alpha_{\mathrm{f}}^{\pi}, 0\right)$ for the $\pi^{\prime}$ polarization in the scattered x-ray. Thereby the RIXS spectra may be expressed as

$$
\begin{aligned}
& W\left(q_{\mathrm{f}}, \boldsymbol{\alpha}_{\mathrm{f}} ; q_{\mathrm{i}}, \boldsymbol{\alpha}_{\mathrm{i}}\right) \\
& =\frac{w^{4}}{4 \omega_{\mathrm{i}} \omega_{\mathrm{f}}}\left(\frac{2}{15}\right)^{2} \times \begin{cases}\left(\alpha_{\mathrm{f}}^{\pi}\right)^{2} Y^{(1)}\left(\omega_{\mathrm{i}} ; \mathrm{q}, \omega\right), & \sigma \rightarrow \pi \\
Y^{(2)}\left(\omega_{\mathrm{i}} ; \mathrm{q}, \omega\right), & \sigma \rightarrow \sigma^{\prime} \\
\left(\alpha_{\mathrm{i}}^{\pi}\right)^{2} Y^{(1)}\left(\omega_{\mathrm{i}} ; \mathrm{q}, \omega\right), & \pi \rightarrow \sigma^{\prime} \\
\left(\alpha_{\mathrm{f}}^{\pi} \alpha_{\mathrm{i}}^{\pi}\right)^{2} Y^{(2)}\left(\omega_{\mathrm{i}} ; \mathrm{q}, \omega\right), & \pi \rightarrow \pi^{\prime}\end{cases}
\end{aligned}
$$

The one-magnon term $Y^{(1)}$ and the two-magnon term $Y^{(2)}$ are separated by the polarization. Accordingly the polarization analysis is useful to clarify the contribution of $Y^{(2)}$. For other directions of q, we could obtain the similar formulas separated by polarizations.

\section{CONCLUDING REMARKS}

We have studied the magnetic excitations on the $L$ edge RIXS from undoped cuprates beyond the FCA. Emphasis is on how the symmetry breaking of the ground state affects the magnetic RIXS spectra. It is found that the spin excitations are brought about at neighbouring sites in addition to the core-hole site. We have shown that the anisotropic terms emerged in the scattering amplitudes as a direct consequence of the broken symmetry. The fact contrasts sharply with the case in neutron scattering, where the amplitude is described through the interaction Hamiltonian between the spins of neutron and electron. The presence of such anisotropic terms has been supported by the calculation on a one-dimensional finitesize ring of spins under the staggered external field and on a two-dimensional cluster with the molecular field acting on the boundary. Collecting up such amplitudes on all the $\mathrm{Cu}$ sites, we have expressed the RIXS spectra in the form of spin correlation functions, which have been calculated within the $1 / S$-expansion. The anisotropic terms have made the RIXS intensity considerably enhanced as $|\mathrm{q}|$ goes to zero. Such enhancement could be confirmed experimentally by observing carefully the spectra around $\mathrm{q}=0$. With a little further improvement on energy resolution, it would make the distinction possible as achieved in $\mathrm{Sr}_{2} \mathrm{IrO}_{4}$, in which band-splitting, predicted by a theory ${ }^{35}$, conspicuous around $\mathrm{q}=(0,0)$ had been discerned by recent experiment ${ }^{36}$. We believe our present emphasize on the anisotropic terms originated from the antiferromagnetic long range order might be insightful when one analyses the systems with short range order such as the doped high- $T_{\mathrm{c}}$ cuprates ${ }^{16-18}$.

\section{ACKNOWLEDGMENTS}

This work was partially supported by a Grant-in-Aid for Scientific Research from the Ministry of Education, Culture, Sports, Science and Technology of the Japanese Government.

\section{Appendix A: Absorption coefficient}

Since the $2 p$ core hole is quite localized in real space, the absorption coefficient is well approximated by the sum of the intensities on each lattice site. Therefore, after averaging the polarization, the absorption coefficient $A\left(j, \omega_{\mathrm{i}}\right)$ at the $\mathrm{Cu} L_{2^{-}}$and $L_{3}$-edges may be expressed as

$$
A\left(j, \omega_{\mathrm{i}}\right) \propto \frac{\Gamma}{\pi} \sum_{\sigma, \eta} \frac{\left|\left\langle\phi_{\eta} \mid \psi_{0}^{\sigma}\right\rangle\right|^{2}}{\left[\omega_{\mathrm{i}}+\epsilon_{\mathrm{g}}-\epsilon_{\mathrm{core}}-\epsilon_{\eta}^{\prime}\right]^{2}+\Gamma^{2}},
$$

where $\epsilon_{\text {core }}$ depends on $j$. By substituting the eigenvalues and the eigenstates evaluated on finite-size clusters into (A1), we obtain $A\left(j, \omega_{\mathrm{i}}\right)$. For $\Gamma$ comparable or larger than the excitation energy $\epsilon_{\eta}^{\prime}, A\left(j, \omega_{\mathrm{i}}\right)$ is close to the Lorentzian curve. The calculated $A\left(j, \omega_{\mathrm{i}}\right)$ for $\Gamma / J=2.4$ in a two-dimensional Heisenberg model has been shown in figure 3 in $\frac{12}{2}$.

\section{Appendix B: Projection onto non-orthogonal bases}

We try to project a state $|F\rangle$ onto the non-orthogonal states $\left|\Psi_{i}\right\rangle$ 's as

$$
|F\rangle=\sum_{i} f_{i}\left|\Psi_{i}\right\rangle
$$

Operating $\left\langle\Psi_{i}\right|$ from the left side of (B1), we obtain $Q_{i}=\sum_{j} \hat{\rho}_{i, j} f_{j}$ where $Q_{i} \equiv\left\langle\Psi_{i} \mid F\right\rangle$ and $\hat{\rho}_{i, j} \equiv\left\langle\Psi_{i} \mid \Psi_{j}\right\rangle$. Therefore the expansion coefficients $f_{i}$ 's are given by $f_{i}=\sum_{j}\left(\hat{\rho}^{-1}\right)_{i, j} Q_{j}$. As long as the number of the projected states remains finite, this procedure uniquely determines the expansion coefficients.

\section{Appendix C: One-dimensional ring of spins under the staggered external field}

We examine how the anisotropic terms develop in concert with the development of the staggered moment. This is achieved easily in one-dimensional system, since there is no long range order in the ground state. To control the staggered moment, we apply the staggered external field. Thereby the Hamiltonian of the system is given by

$$
H_{\mathrm{mag}}=J \sum_{\langle i, j\rangle} \mathrm{S}_{i} \cdot \mathrm{S}_{j}+H_{\mathrm{ex}} \sum_{i}(-1)^{i} S_{i}^{z^{\prime}},
$$

where the field strength is denoted as $H_{\mathrm{ex}}$. We consider a system made of 12 spins of $S=\frac{1}{2}$ with periodic boundary condition, as shown in figure 8

Since the total-spin component along the $z^{\prime}$ axis is zero in the ground state, the Hamiltonian matrix is represented by a matrix with $924 \times 924$ dimensions. Diagonalizing the Hamiltonian matrix, we obtain the ground-state wavefunction. The inset in figure 9 (a) shows the staggered magnetization $\left\langle S_{0}^{z^{\prime}}\right\rangle$ as a function of $H_{\mathrm{ex}}$, which 


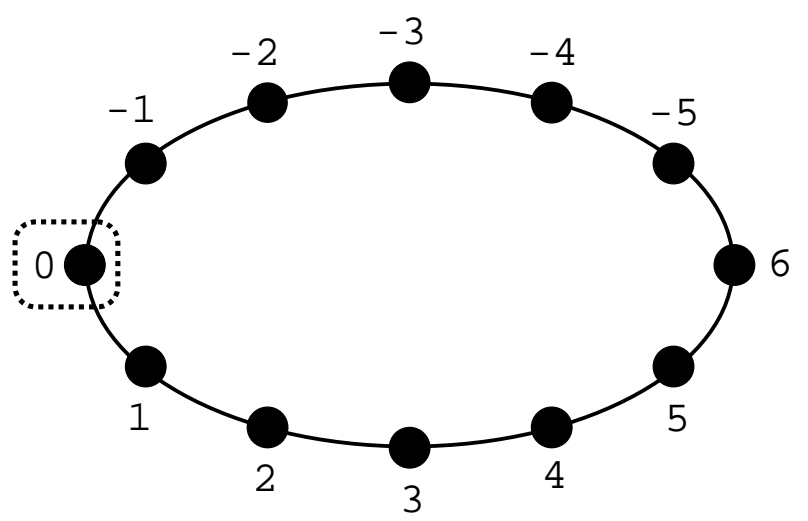

FIG. 8: A ring of 12 spins $\left(S=\frac{1}{2}\right)$ used to evaluate the coefficients for the spin excitations in RIXS. The spin at site 0 is annihilated in the intermediate state.

TABLE II: Coefficients for isotropic terms in units of $1 / J$ in the one-dimensional ring of 12 spins $\left(S=\frac{1}{2}\right)$. The incident photon energy $\omega_{\mathrm{i}}$ is set to give the maximum absorption coefficient.

\begin{tabular}{cccc}
\hline \hline$\Gamma / J$ & $f_{0}^{(1)}\left(\omega_{\mathrm{i}}\right)$ & $f_{1}^{(1)}\left(\omega_{\mathrm{i}}\right)$ & $f_{2}^{(2)}\left(\omega_{\mathrm{i}}\right)$ \\
\hline 2.0 & $(-0.013,-0.500)$ & $(0.043,-0.025)$ & $(0.366,-0.219)$ \\
1.5 & $(0.025,-0.672)$ & $(0.088,-0.051)$ & $(0.633,-0.365)$ \\
1.0 & $(0.050,-1.009)$ & $(0.154,-0.125)$ & $(1.101,-0.894)$ \\
\hline \hline
\end{tabular}

increases with increasing $H_{\text {ex }}$. The intermediate state, on the other hand, consists of 11 spins, since the spin degree of freedom is lost at the core-hole site. Thereby the Hamiltonian matrix in the intermediate state is represented by a matrix with $462 \times 462$ dimensions in the subspace of $\sum_{i} S_{i}^{z^{\prime}}= \pm \frac{1}{2}$. Using the eigenvalues and eigenfunctions for the Hamiltonian of the intermediate state together with the ground state, we evaluate (3.11), (3.19), and others for the coefficients of the spin excitations.

By setting $H_{\mathrm{ex}}=0$, we first evaluate the isotropic terms in the absence of the anisotropic terms. Table II shows the coefficients for isotropic terms for several values of $\Gamma / J$ with $\omega_{\mathrm{i}}$ being fixed at the value to give the maximum absorption coefficient. The values $\Gamma / J=2.0$ and 1.5 may correspond to $\mathrm{CaCu}_{2} \mathrm{O}_{3} 37$ and $\mathrm{Sr}_{2} \mathrm{CuO}_{3}{ }^{38}$, respectively. The coefficient $f_{1}^{(1)}\left(\omega_{\mathrm{i}}\right)$ for the spin-flip excitation on neighbouring sites is much smaller than $f_{0}^{(1)}\left(\omega_{\mathrm{i}}\right)$. The coefficient $f_{2}^{(2)}\left(\omega_{\mathrm{i}}\right)$ for the $\mathrm{S}_{0} \cdot \mathrm{X}$ term is comparable to the coefficient $f_{0}^{(1)}\left(\omega_{\mathrm{i}}\right)$ for the spin-flip term. It grows with decreasing $\Gamma / J$, as was discussed in 13 .

Next, we turn our attention to the anisotropic terms. Figures 9(b) and (c) show the absolute values of the coefficients as a function of staggered moment for $\Gamma / J=2.0$. They demonstrate that the anisotropic terms develop with increasing staggered moment as expected. Note that the magnitudes of the isotropic terms vary gradually and slightly diminish rather than increase with increasing

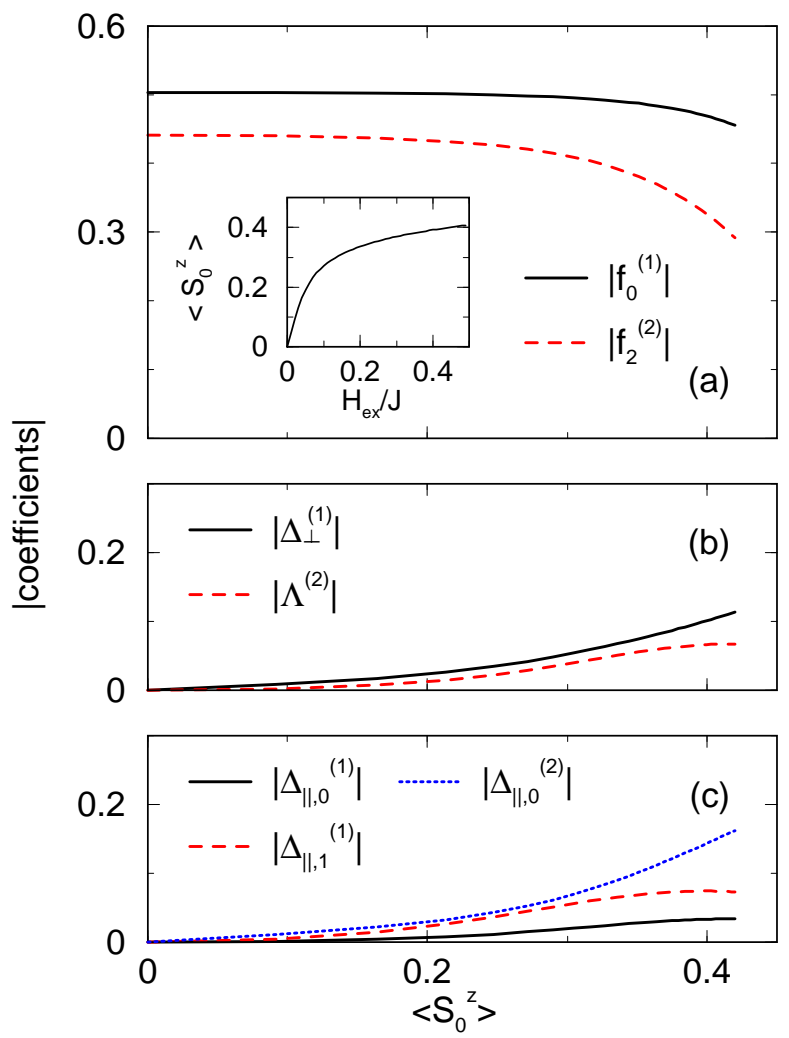

FIG. 9: Various coefficients measured in units of $J^{-1}$ as a function of the staggered moment evaluated in a ring of 12 spins. $\Gamma / J=2.0$, and $\omega_{\mathrm{i}}$ is set to give rise to the maximum absorption coefficient. (a) The (black) solid and (red) broken curves correspond to $\left|f_{0}^{(1)}\right|$ and $\left|f_{2}^{(2)}\right|$, respectively. (b) The (black) solid and (red) broken curves represent $\left|\Delta_{\perp}^{(1)}\right|$ and $\left|\Lambda^{(2)}\right|$, respectively. (c) The (black) solid, (red) broken, and (blue) dotted curves show $\left|\Delta_{\|, 0}^{(1)}\right|,\left|\Delta_{\|, 1}^{(1)}\right|$, and $\left|\Delta_{\|, 0}^{(2)}\right|$, respectively. The inset in Panel (a) shows the staggered moment as a function of $H_{\mathrm{ex}} / J$.

staggered moment as shown in figure 9 (a).

\section{Appendix D: $1 / S$-expansion}

Here, we briefly summarise an introduction of the $1 / S$ expansion. The emphasis is on the definitions of the quantities used in the main text. The details are relegated to the references such as $\frac{12}{2}$. Assuming two sublattices in the antiferromagnetic ground state, we express spin operators by boson operators $\operatorname{as}^{24}$

$$
\begin{aligned}
S_{i}^{z} & =S-a_{i}^{\dagger} a_{i}, \\
S_{i}^{+} & =\left(S_{i}^{-}\right)^{\dagger}=\sqrt{2 S} f_{i}(S) a_{i}, \\
S_{j}^{z} & =-S+b_{j}^{\dagger} b_{j}, \\
S_{j}^{+} & =\left(S_{j}^{-}\right)^{\dagger}=\sqrt{2 S} b_{j}^{\dagger} f_{j}(S),
\end{aligned}
$$


where $a_{i}$ and $b_{j}$ are boson annihilation operators, and

$$
f_{\ell}(S)=\sqrt{1-\frac{n_{\ell}}{2 S}}=1-\frac{1}{2} \frac{n_{\ell}}{2 S}-\frac{1}{8}\left(\frac{n_{\ell}}{2 S}\right)^{2}+\cdots,
$$

with $n_{\ell}$ represents $a_{i}^{\dagger} a_{i}$ and $b_{j}^{\dagger} b_{j}$ for $\ell=i$ and $j$, respectively. Indices $i$ and $j$ refer to sites on the A and $\mathrm{B}$ sublattices, respectively. Using (D1)-(D4), $H_{\text {mag }}$ is expanded in powers of $1 / S$,

$$
H_{\mathrm{mag}}=-J S^{2} N z / 2+H_{\mathrm{mag}}^{(0)}+H_{\mathrm{mag}}^{(1)}+\cdots,
$$

where $N$ and $z$ are the number of lattice sites and that of nearest neighbour sites, respectively. $H_{\text {mag }}^{(n)}$ stands for the $n$-th order term in the $1 / S$-expansion. The Fourier transforms of the boson operators are introduced within the first MBZ,

$$
a_{i}=(2 / N)^{\frac{1}{2}} \sum_{\mathrm{k}} a_{\mathrm{k}} \mathrm{e}^{\mathrm{i} k \cdot \mathrm{r}_{\mathrm{i}}}, b_{j}=(2 / N)^{\frac{1}{2}} \sum_{\mathrm{k}} b_{\mathrm{k}} \mathrm{e}^{\mathrm{i} k \cdot \mathrm{r}_{\mathrm{j}}} .
$$

Then, with the help of a Bogoliubov transformation,

$$
a_{\mathrm{k}}^{\dagger}=\ell_{\mathrm{k}} \alpha_{\mathrm{k}}^{\dagger}+m_{\mathrm{k}} \beta_{-\mathrm{k}}, \quad b_{-\mathrm{k}}=m_{\mathrm{k}} \alpha_{\mathrm{k}}^{\dagger}+\ell_{\mathrm{k}} \beta_{-\mathrm{k}},
$$

we could diagonalize $H_{\text {mag }}^{(0)}$ as

$$
H_{\mathrm{mag}}^{(0)}=J S z \sum_{\mathrm{k}}\left(\epsilon_{\mathrm{k}}-1\right)+J S z \sum_{\mathrm{k}} \epsilon_{\mathrm{k}}\left(\alpha_{\mathrm{k}}^{\dagger} \alpha_{\mathrm{k}}+\beta_{\mathrm{k}}^{\dagger} \beta_{\mathrm{k}}\right) .
$$

Here,

$$
\begin{array}{ll}
\ell_{\mathrm{k}}=\sqrt{\frac{1+\epsilon_{\mathrm{k}}}{2 \epsilon_{\mathrm{k}}}}, \quad m_{\mathrm{k}}=-\sqrt{\frac{1-\epsilon_{\mathrm{k}}}{2 \epsilon_{\mathrm{k}}}}=-x_{\mathrm{k}} \ell_{\mathrm{k}},(\mathrm{D} 10) \\
\epsilon_{\mathrm{k}}=\sqrt{1-\gamma_{\mathrm{k}}^{2}}, \quad \gamma_{\mathrm{k}}=\frac{1}{z} \sum_{\delta} \exp (\mathrm{ik} \cdot \boldsymbol{\delta}), \quad(\mathrm{D} 11)
\end{array}
$$

where $\boldsymbol{\delta}$ connects the origin with the nearest neighbour sites. The expression for $H_{\text {mag }}^{(1)}$ becomes 39

$$
\begin{aligned}
& H_{\mathrm{mag}}^{(1)}=\frac{J S z}{2 S} A \sum_{\mathrm{k}} \epsilon_{\mathrm{k}}\left(\alpha_{\mathrm{k}}^{\dagger} \alpha_{\mathrm{k}}+\beta_{\mathrm{k}}^{\dagger} \beta_{\mathrm{k}}\right) \\
& +\frac{-J S z}{2 S N} \sum_{\mathrm{k}_{1}, \mathrm{k}_{2}, \mathrm{k}_{3}, \mathrm{k}_{4}} \delta_{\mathrm{G}}\left(\mathrm{k}_{1}+\mathrm{k}_{2}-\mathrm{k}_{3}-\mathrm{k}_{4}\right) \\
& \times \ell_{\mathrm{k}_{1}} \ell_{\mathrm{k}_{2}} \ell_{\mathrm{k}_{3}} \ell_{\mathrm{k}_{4}}\left(4 \alpha_{\mathrm{k}_{1}}^{\dagger} \beta_{-\mathrm{k}_{4}}^{\dagger} \beta_{-\mathrm{k}_{2}} \alpha_{\mathrm{k}_{3}} B_{\mathrm{k}_{1} \mathrm{k}_{2} \mathrm{k}_{3} \mathrm{k}_{4}}^{(3)}+\cdots\right),
\end{aligned}
$$

with $A=\frac{2}{N} \sum_{\mathrm{k}}\left(1-\epsilon_{\mathrm{k}}\right)^{30}$. For the square lattice, $A=0.1579$. The Kronecker delta $\delta_{\mathrm{G}}\left(\mathrm{k}_{1}+\mathrm{k}_{2}-\mathrm{k}_{3}-\mathrm{k}_{4}\right)$ indicates the conservation of momenta within a reciprocal lattice vector G. In the second term of (D12), only the relevant term representing scattering of two magnons is shown explicitly. The vertex function $B^{(3)}$ in a symmetric parametrization as well as omitted terms are found in $31,39,40$.
1 L. J. P. Ament, M. van Veenendaal, T. P. Devereaux, J. P. Hill, and J. van den Brink, Rev. Mod. Phys. 83, 705 (2011).

${ }^{2}$ L. Braicovich, L. J. P. Ament, V. Bisogni, F. Forte, C. Aruta, G. Balestrino, N. B. Brookes, G. M. De Luca, P. G. Medaglia, F. M. Granozio, et al., Phys. Rev. Lett. 102, 167401 (2009).

3 L. Braicovich, J. van den Brink, V. Bisogni, M. M. Sala, L. J. P. Ament, N. B. Brookes, G. M. De Luca, M. Salluzzo, T. Schmitt, V. N. Strocov, et al., Phys. Rev. Lett. 104, 077002 (2010).

4 M. Guarise, B. D. Piazza, M. M. Sala, G. Ghiringhelli, L. Braicovich, H. Berger, J. N. Hancock, D. van der Marel, T. Schmitt, V. N. Strocov, et al., Phys. Rev. Lett. 105, 157006 (2010).

5 M. L. Tacon, G. Ghiringhelli, J. Chaloupka, M. M. Sala, V. Hinkov, M. W. Haverkort, M. Minola, M. Bakr, K. J. Zhou, S. Blanco-Canosa, et al., Nat. Mater. 7, 725 (2011).

${ }^{6}$ M. P. M. Dean, R. S. Springell, C. Monney, K. J. Zhou, J. Pereiro, I. Božović, B. D. Piazza, H. M. Rønnow, E. Morenzoni, J. van den Brink, et al., Nat. Mater. 11, 850 (2012).

7 M. P. M. Dean, J. Magn. Magn. Mater. 376, 3 (2015).

8 G. Ghiringhelli, A. Piazzalunga, C. Dallera, T. Schmitt, V. N. Strocov, J. Schlappa, L. Patthey, X. Wang, H. Berger, and M. Grioni, Phys. Rev. Lett. 102, 027401 (2009).

9 K. J. Zhou, Y. B. Huang, C. Monney, X. Dai, V. N. Strocov, N. L. Wang, Z. G. Chen, C. Zhang, P. Dai, L. Patthey, et al., Nat. Commun. 4, 1470 (2013).
10 J. Kim, D. Casa, M. H. Upton, T. Gog, Y.-J. Kim, J. F. Mitchell, M. van Veenendaal, M. Daghofer, J. van den Brink, G. Khaliullin, et al., Phys. Rev. Lett. 108, 177003 (2012).

11 J. Kim, A. H. Said, D. Casa, M. H. Upton, T. Gog, M. Daghofer, G. Jackeli, J. van den Brink, G. Khaliullin, and B. J. Kim, Phys. Rev. Lett. 109, 157402 (2012).

12 J. I. Igarashi and T. Nagao, Phys. Rev. B 85, 064421 (2012).

13 J. I. Igarashi and T. Nagao, Phys. Rev. B 85, 064422 (2012).

14 S. Kourtis, J. van den Brink, and M. Daghofer, Phys. Rev. B 85, 064423 (2012).

15 T. Nagao and J. I. Igarashi, Phys. Rev. B 85, 224436 (2012).

16 W. Chen and O. P. Sushkov, Phys. Rev. B 88, 184501 (2013).

17 C. J. Jia, E. A. Nowadnick, K. Wholfeld, Y. F. Kung, C. C. Chen, S. Johnston, T. Tohyama, B. Moritz, and T. P. Devereaux, Nat. Commun. 5, 3314 (2014).

18 D. Benjamin, I. Klich, and E. Demler, Probing competing and intertwined orders with resonant inelastic x-ray scattering in the hole-doped cuprates (2014), arXiv:1407.3843.

19 G. Ghiringhelli, N. B. Brookes, E. Annese, H. Berger, C. Dallera, M. Grioni, L. Perfetti, A. Tagliaferri, and L. Braicovich, Phys. Rev. Lett. 92, 117406 (2004).

${ }^{20}$ L. J. P. Ament, G. Ghiringhelli, M. M. Sala, L. Braicovich, and J. van den Brink, Phys. Rev. Lett. 103, 117003 (2009).

21 M. W. Haverkort, Phys. Rev. Lett. 105, 167404 (2010). 
${ }^{22}$ V. Bisogni, S. Kourtis, C. Monney, K. Zhou, R. Kraus, C. Sekar, V. Strocov, B. Bucher, J. van den Brink, L. Braicovich, et al., Phys. Rev. Lett. 112, 147401 (2014).

23 V. Bisogni, L. Simonelli, L. J. P. Ament, F. Forte, M. Moretti, M. Minola, S. Huotari, J. van den Brink, G. Ghiringhelli, N. B. Brookes, et al., Phys. Rev. B 85, 214527 (2012).

24 T. Holstein and H. Primakoff, Phys. Rev. 58, 1098 (1940).

25 T. Tonegawa, Prog. Theor. Phys. 40, 1195 (1968).

26 C. C. Wan, A. B. Harris, and D. Kumar, Phys. Rev. B 48, 1036 (1993).

27 M. E. Rose, Elementary Theory of Angular Momentum (John Wiley \& Sons Inc., 1957).

28 Notice that, in our previous paper ${ }^{12}$, the symbol $f_{n}^{(1)}\left(\omega_{\mathrm{i}}\right)$ instead of $g_{n}^{(1)}\left(\omega_{\mathrm{i}}\right)$ was used since they are equivalent when the anisotropic terms are neglected or in the absence of long-range order.

29 D. Vaknin, S. K. Sinha, D. E. Moncton, D. C. Johnston, J. M. Newsam, C. R. Safinya, and H. E. King Jr., Phys. Rev. Lett. 58, 2802 (1987).

30 T. Oguchi, Phys. Rev. 117, 117 (1960).

31 J. I. Igarashi, Phys. Rev. B 46, 10763 (1992).
32 T. Nagao and J. I. Igarashi, Phys. Rev. B 75, 214414 (2007).

33 J. P. Hill, G. Blumberg, Y. -J. Kim, D. S. Ellis, S. Wakimoto, R. J. Birgeneau, S. Komiya, Y. Ando, B. Liang, R. L. Greene, et al., Phys. Rev. Lett. 100, 097001 (2008).

${ }^{34}$ F. Forte, L. J. P. Ament, and J. van den Brink, Phys. Rev. B 77, 134428 (2008).

35 J. I. Igarashi and T. Nagao, Phys. Rev. B 88, 104406 (2013).

36 J. Kim, M. Daghofer, A. H. Said, T. Gog, J. van den Brink, G. Khaliullin, and B. J. Kim, Nat. Commun. 5, 4453 (2014).

37 B. Lake, A. M. Tsvelik, S. Notbohm, D. A. Tennant, T. G. Perring, M. Reehuis, C. Sekar, G. Krabbes, and B. Büchner, Nat. Phys. 6, 50 (2010).

38 A. C. Walters, T. G. Perring, J.-S. Caux, A. T. Savici, G. D. Gu, C.-C. Lee, W. Ku, and I. A. Zaliznyak, Nat. Phys. 5, 867 (2009).

39 A. B. Harris, D. Kumar, B. I. Halperin, and P. C. Hohenberg, Phys. Rev. B 3, 961 (1971).

40 A. V. Syromyatnikov, J. Phys.: Condens. Matter 22, 216003 (2010). 